\title{
Intrabody-Induced Cell Death by Targeting the T. brucei Cytoskeletal Protein TbBILBO1
}

\author{
Christine E. Broster Reix, ${ }^{a}$ Miharisoa Rijatiana Ramanantsalama, ${ }^{a}$ Carmelo Di Primo, ${ }^{b}$ Laëtitia Minder, ${ }^{\text {b,c }}$ Mélanie Bonhivers, ${ }^{a}$ \\ Denis Dacheux, ${ }^{\mathrm{a}, \mathrm{d}}$ (D) Derrick R. Robinson ${ }^{\mathrm{a}}$
}

aUniversity of Bordeaux, CNRS, Microbiologie Fondamentale et Pathogénicité, UMR 5234, Bordeaux, France

bUniversity of Bordeaux, INSERM U1212-CNRS UMR 5320, ARNA laboratory, European Institute of Chemistry and Biology, Pessac cedex, France

CUniversity of Bordeaux, CNRS UMS3033-INSERM US001, European Institute of Chemistry and Biology, Pessac cedex, France

dBordeaux INP, Microbiologie Fondamentale et Pathogénicité, UMR 5234, Bordeaux, France

Christine E. Broster Reix and Miharisoa Rijatiana Ramanantsalama contributed equally to this article. Author order was determined alphabetically.

ABSTRACT Trypanosoma brucei belongs to a genus of protists that cause life-threatening and economically important diseases of human and animal populations in Sub-Saharan Africa. T. brucei cells are covered in surface glycoproteins, some of which are used to escape the host immune system. Exo-/endocytotic trafficking of these and other molecules occurs via a single copy organelle called the flagellar pocket (FP). The FP is maintained and enclosed around the flagellum by the flagellar pocket collar (FPC). To date, the most important cytoskeletal component of the FPC is an essential calcium-binding, polymer-forming protein called TbBILBO1. In searching for novel tools to study this protein, we raised nanobodies ( $\mathrm{Nb}$ ) against purified, full-length TbBILBO1. Nanobodies were selected according to their binding properties to TbBILBO1, tested as immunofluorescence tools, and expressed as intrabodies (INb). One of them, $\mathrm{Nb} 48$, proved to be the most robust nanobody and intrabody. We further demonstrate that inducible, cytoplasmic expression of INb48 was lethal to these parasites, producing abnormal phenotypes resembling those of TbBILBO1 RNA interference (RNAi) knockdown. Our results validate the feasibility of generating functional single-domain antibody-derived intrabodies to target trypanosome cytoskeleton proteins.

IMPORTANCE Trypanosoma brucei belongs to a group of important zoonotic parasites. We investigated how these organisms develop their cytoskeleton (the internal skeleton that controls cell shape) and focused on an essential protein (BILBO1) first described in $T$. brucei. To develop our analysis, we used purified BILBO1 protein to immunize an alpaca to make nanobodies $(\mathrm{Nb})$. Nanobodies are derived from the antigen-binding portion of a novel antibody type found only in the camel and shark families of animals. Anti-BILBO1 nanobodies were obtained, and their encoding genes were inducibly expressed within the cytoplasm of $T$. brucei as intrabodies (INb). Importantly, INb48 expression rapidly killed parasites producing phenotypes normally observed after RNA knockdown, providing clear proof of principle. The importance of this study is derived from this novel approach, which can be used to study neglected and emerging pathogens as well as new model organisms, especially those that do not have the RNAi system.

KEYWORDS BILBO1, cytoskeleton, intrabody, nanobody, parasite, trypanosoma

rypanosomes are flagellated protists comprised of pathogenic species capable of infecting a wide range of vertebrate hosts, including humans and domestic and wild animals. Trypanosomes cause lethal and economically important human and animal diseases worldwide (1, 2). A limited number of diagnostic tests and treatments are available for human and animal African trypanosomiasis. Fortunately, the first oral trypanocidal drug, fexinidazole,
Citation Broster Reix CE, Ramanantsalama MR, Di Primo C, Minder L, Bonhivers M, Dacheux D, Robinson DR. 2021. Intrabody-induced cell death by targeting the $T$. brucei cytoskeletal protein TbBILBO1. Microbiol Spectr 9:e00915-21. https://doi.org/10.1128/Spectrum.00915-21. Editor Vasant Muralidharan, University of Georgia

Copyright $\odot 2021$ Broster Reix et al. This is an open-access article distributed under the terms of the Creative Commons Attribution 4.0 International license.

Address correspondence to Derrick R. Robinson, derrick-roy.robinson@u-bordeaux.fr.

Received 16 July 2021

Accepted 30 September 2021

Published 27 October 2021 
was recently approved for use in humans in Africa, which simplifies the treatment regimen for certain cases $(3,4)$. However, millions of wild animals and domesticated livestock are still at risk of infection, while the economic burden of the disease to the African economy is estimated to be 4.7 billion U.S. dollars (USD) per year due to the death of around 3 million head of cattle (5) (http://www.fao.org/paat/the-programme/the-disease/en/). Understanding the basic biology of any virulent organism is important to fully understand its pathogenicity. Furthermore, new targets, tools, and models are needed.

Trypanosomes possess a highly organized cytoskeleton comprised primarily of microtubules but also many cytoskeleton-associated proteins (5-12). Most pathogenic trypanosomes possess a single copy organelle called the flagellar pocket (FP) at the site where the flagellum exits the cell body, which is physically connected to the cytoskeleton (12-17). In many species, the FP is the sole site of exo- and endocytosis. In Trypanosoma brucei, the FP is essential for survival and has a cytoskeleton component called the flagellar pocket collar (FPC) that circumvents the neck of the FP at the site where the flagellum exits the cell $(13,14,18,19)$. The FPC forms a cytoskeletal boundary or interface at the site of contact between the pellicular, flagellar, and FP membranes $(13,20,21)$. To date, only a few FPC and FPC-associated proteins have been identified and only two essential proteins have been characterized. The first is TbBILBO1 (67 kDa; 587 amino acids [aa]), which is the main protein component of the flagellar pocket collar (FPC), and the second is TbMORN1 (40.9 kDa; $358 \mathrm{aa),} \mathrm{which} \mathrm{is} \mathrm{present} \mathrm{in} \mathrm{the} \mathrm{hook} \mathrm{com-}$ plex $(\mathrm{HC})$, a structure intimately linked and immediately distal to the FPC, and is thought to indirectly play a role in facilitating protein entry into the cell. Two other FPC and FPC-associated proteins, $\mathrm{TbBILBO} 2$ and TbFPC4, respectively, are TbBILBO1 binding partners involved in a tripartite interaction $(21,22)$.

The $\mathrm{N}$ terminus of TbBILBO1 adopts a ubiquitin-like fold, followed by two calcium-binding EF-hand domains (EFh1: amino acid residues 185 to 213 and EFh2 221 to 249). These domains are followed by a large coiled-coil domain (CC; amino acids 263 to 566) and a C-terminal leucine zipper (LZ; amino acid residues from 534 to 578) that are involved in dimerization and polymerization, respectively $(20,22-25)$. RNA interference (RNAi)-mediated knockdown of TbBILBO1 in the cultured, insect procyclic form (PCF) parasites prevents FPC and FP biogenesis, induces the aberrant repositioning of the new flagellum (detached from the cell body along its length), and results in parasite cell death. Knockdown of TbBILBO1 in the mammalian bloodstream form (BSF) is rapidly lethal (13). The FPC is also intimately associated with the microtubule quartet (MtQ), a subset of specialized microtubules that originate near the basal bodies of the flagellum and grow very close to, if not through, the FPC $(22,26,27)$. Previous published work has identified binding of TbMORN1 and TbSPEF1 (a microtubule binding and bundling protein) onto detergent and salt-extracted MtQ, and prior to those studies, our anti-TbBILBO1 (IgM) antibody demonstrated both an FPC and a minor MtQ signal $(13,27,28)$.

We searched for novel, easily modifiable molecular and immunological tools to study BILBO1 and decided to raise nanobodies against TbBILBO1. Nanobodies are derived from a specific class of heavy chain only antibodies (HCAb) found naturally in Camelidae (alpacas, camels, llamas, and vicunas) and also in Elasmobranchs (cartilaginous fish: sharks and rays) $(29,30)$. Nanobodies are recombinant antibody fragments derived from the antigen-binding domain (variable domain, $\mathrm{VHH}$ ) of heavy chain antibodies and are much smaller than traditional antibodies, being $\sim 15 \mathrm{kDa}$ in mass (31-33). The use of nanobodies is greatly expanding in cellular and molecular biology, diagnostics, and medicine. Indeed, in 2018, caplacizumab was approved as the first nanobody-based drug (34). Intrabodies (INbs), cytoplasmic nanobodies, have many potential roles ranging from pathogen detection to killing tumor cells, as well as therapeutic use (35-38). Numerous studies have used nanobodies to characterize a wide variety of proteins, but to date, only one nanobody has been developed against a trypanosome cytoskeleton protein, (Nb392), targeting the paraflagellar rod protein (PFR1) (39).

RNA interference (RNAi) has been used to greatly enhance knowledge of gene and consequently protein function in living cells. However, RNAi can have several limitations, such as incomplete knockdown of the target protein. Furthermore, the double-stranded 
RNA produced during RNAi has a relatively short half-life compared to that of an intrabody. Intrabodies are nanobodies expressed cytoplasmically in the target cell and have the benefit of having high antigen specificity, the ability to recognize and bind to a structural epitope not accessible by conventional antibodies, and potential as tools for many biological systems (40-43). One advantage of intrabodies is that they act at a posttranslation stage and therefore can target a specific isoform or posttranslational modification of a protein (43). Also, in the case of essential or structural proteins, induction of the expression of INbs at a specific cell cycle, life cycle stage, or point in organelle biogenesis may provide very subtle and precise phenotypes. In this regard, nanobodies also have advantages over conventional antibodies due to their smaller size, allowing access to cryptic epitopes and ability to cross the blood-brain barrier $(44,45)$. Conventional antibodies have a further disadvantage of multi-epitope cross-reaction, a phenomenon exemplified by $\mathrm{lgE}$ in allergen cross-reaction, whereas nanobodies are, theoretically, single epitope specific $(46,47)$.

We show here that our anti-TbBILBO1 (aa 1-110) rabbit polyclonal, purified Nb48, and intrabody 48 (INb48) all bind to the FPC and the MtQ. Importantly, we demonstrate that intrabodies raised against TbBILBO1 can have effects comparable to RNAi knockdown and we establish that anti-TbBILBO1 intrabody (INb48) identifies its target protein when expressed in a homologous mammalian system in vitro and within the cytoplasm of $T$. brucei. Our data illustrate, for the first time, a functional anti-cytoskeletal intrabody in T. brucei that is able to target and bind precisely to its target protein epitope and block biogenesis of a cytoskeletal structure leading to rapid cell death. These data support the hypothesis that targeting minor, yet essential, cytoskeletal proteins is of considerable merit in the search to understand parasite biology. The results also suggest that the use of intrabodies might be useful in organisms that do not have RNAi machinery or to characterize the cell biology of emerging pathogens, marine organisms, protists, and the numerous new model organisms such as those described by Faktorová et al. (48).

\section{RESULTS}

Production of anti-TbBILBO1 nanobodies. Full-length histidine-tagged TbBILBO1 protein was expressed in bacteria and then used to immunize an alpaca to produce TbBILBO1-targeting nanobodies by the Nanobody Service Facility (NSF, VIB, Brussels). Seven different nanobodies (Nbs) were identified following phage display screening. These were assigned to three different groups based on their amino acid sequences. It was hypothesized that $\mathrm{Nbs}$ in the same group would recognize the same epitope, but their other characteristics, such as affinity, potency, stability, and expression yield, could be different. We chose to focus on one nanobody from each group for our current studies: $\mathrm{Nb} 48, \mathrm{Nb9}$, and $\mathrm{Nb73}$.

Nb48 as a functional tool for immunolabelling TbBILBO1. The Nbs were cloned in the pHENC6c vector in frame with a hemagglutinin ( $\mathrm{HA})$ and histidine tag ( $\mathrm{Nb}_{\text {:HA:6His }}$ ), allowing the secretion of the Nbs in the periplasm, their purification using metal affinity chromatography, and their detection (Fig. S1). Samples of noninduced (-) and induced bacteria $(+)$, pellet $(P)$, and periplasmic extracts (Ex) were probed by Western blotting with antiHA (Fig. S2A and B). For simplicity, the HA::6His-tagged nanobodies are hereafter called $\mathrm{Nb} 48, \mathrm{Nb9}$, and $\mathrm{Nb73}$ (with tags written in subscript). We tested purified Nb48::HA:6His' Nb9:-HA:6His' and Nb73:HA:6His by using anti-HA for detection in immunofluorescence microscopy (IFA) on T. brucei wild-type (WT) procyclics (PCF) detergent-extracted cells (cytoskeletons) (Fig. 1). For convenience, in Figures 1 and 2, the cells that are probed with purified nanobodies are labeled "Anti-Nb," plus the relevant nanobody name. In these experiments, purified $\mathrm{Nb48}:$ :HA:6His labels endogenous TbBILBO1 at the FPC, as seen by colocalization with antiTbBILBO1 (polyclonal antibody 1-110) (Fig. 1A). Nb9 ${ }_{\text {:HA:6His }}$ does not label wild-type cytoskeletons expressing endogenous TbBILBO1 protein (Fig. 1B) but does label TbBILBO1 in cells that overexpress TbBILBO1 $1_{: 3 \mathrm{cmyc}}$ (Fig. 1C). Importantly, Nb73:HA:6His was negative in our experiments and did not label TbBILBO1 in either endogenous (Fig. 1D) or overexpressed levels of TbBILBO1 (not shown). To exclude the possibility of cross-reaction between primary and/ or secondary antibodies in double labeling protocols, $\mathrm{Nb} 8_{\text {:HA:6His }}$ and anti-TbBILBO1 (1-110) were also used alone to probe cytoskeletons. In both cases, the subsequent labeling was 


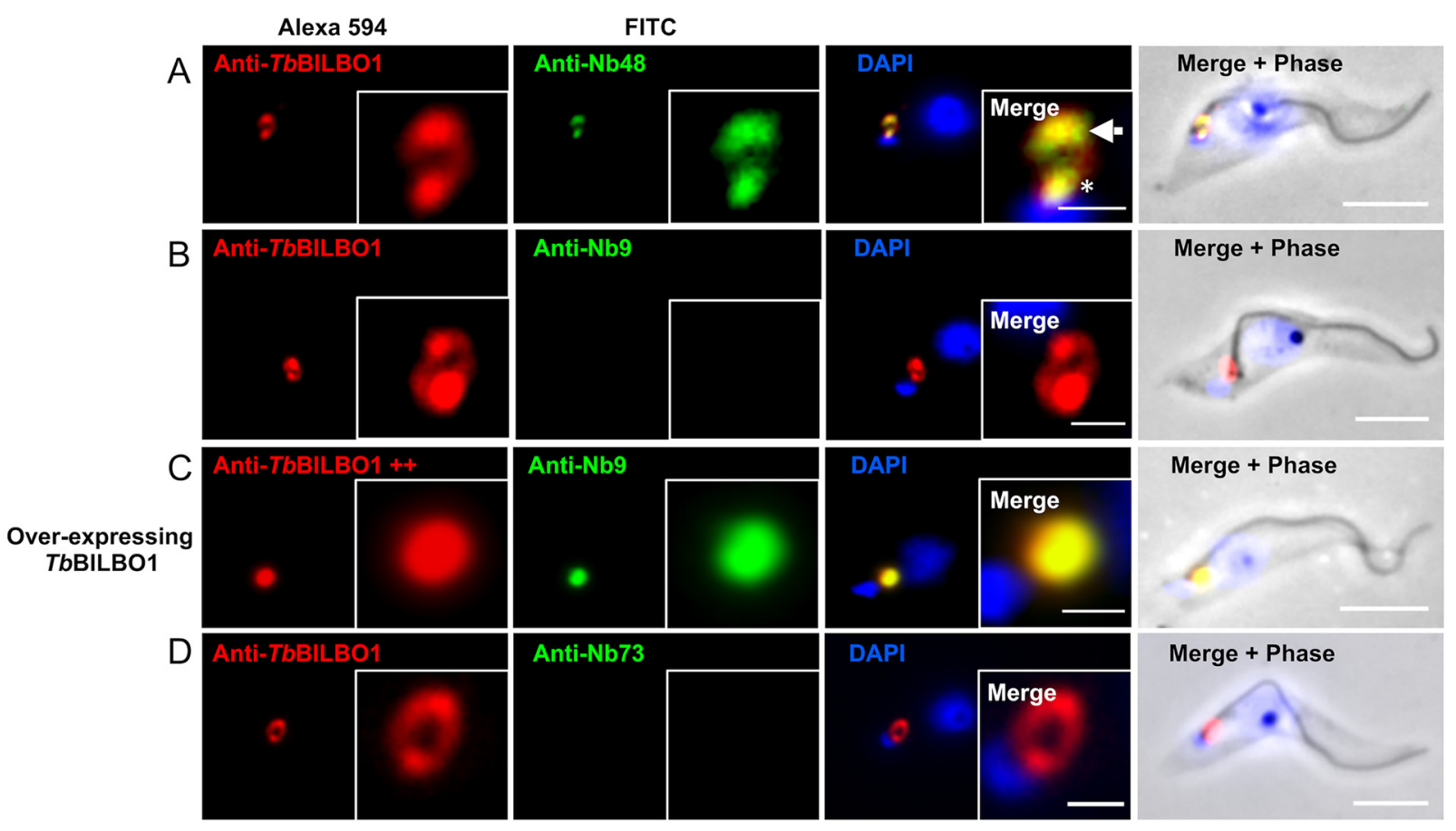

FIG 1 Nanobodies as TbBILBO1 immunofluorescence detection tools. (A) IFA testing of purified Nb48 brucei cytoskeletons showing colocalization with TbBILBO1 (probed with anti-TbBILBO1 aa 1-110 rabbit; red). The arrow indicates the FPC and the asterisk indicates colabeling on the MTQ; both of these structures are colabeled. (B) Purified Nb9 ${ }_{\text {:HA:6His }}$ does not label endogenous TbBILBO1 in WT cytoskeletons. (C) Nb9: $9_{\text {HA: } 6 \mathrm{His}}$ on T. brucei overexpressing TbBILBO1 (++) shows colocalization with TbBILBO1. (D) Purified Nb73::HA:6His on WT cytoskeletons does not label TbBILBO1. Scale bar is $5 \mu \mathrm{m}$, inset is $1 \mu \mathrm{m}$. For the sake of convenience, the proteins being probed in all figures are named anti-Nb, plus the relevant nanobody name rather than its tag.

observed on the FPC and the microtubule quartet (MtQ), a subset of specialized microtubules that are in close proximity to the FPC, but with unknown function (27) (Fig. 2A and B). Labeling with anti-TbBILBO1 (rabbit polyclonal, 1-110) alone also clearly labeled the MtQ (Fig. 2C). Colocalization was observed in double labeling experiments with anti-TbBILBO1 (1-110) and Nb48:HA:6His (Fig. $1 \mathrm{~A}$ and Fig. 2D). In Figures $2 \mathrm{~A}$ to $\mathrm{D}$, the asterisks indicate the labeling of the MtQ. Purified $\mathrm{Nb} 48_{\text {:HA:6His }}$ and $\mathrm{Nb9}{ }_{\text {:HA:6His }}$ both positively label full-length TbBILBO1 protein from T. brucei whole-cell extracts in Western blotting experiments (Fig. 2E and $\mathrm{F}$ ) as well as TbBILBO1 truncated versions (T3 $3_{: 3 \mathrm{cMyc}}$ aa 171 to 587, and T4: $4_{\mathrm{zcMyc}}$ aa 251 to 587; please also refer to the schematic in Figure $2 \mathrm{G}$ for an overview of the truncations).

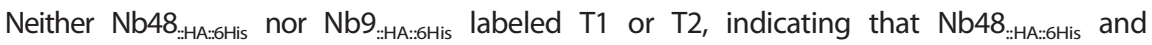
$\mathrm{Nb9}$ :HA: $6 \mathrm{His}_{\text {is }}$ bind to TbBILBO1 in the region ranging from aa 251 to aa 587. Controls for IFA experiments in Figures 1 and 2 are presented in Figure S2C.

Surface plasmon resonance confirms a strong binding affinity of Nb48 to TbBILB01. To determine the equilibrium dissociation constant $\left(K_{D}\right)$ of $\mathrm{Nb48}: \mathrm{HA:6 \textrm {His }}$ (group 1) and $\mathrm{Nb9}$ ::HA::6His (group 2) to TbBILBO1, we used surface plasmon resonance (SPR) (Fig. 2H and I) (note: it was not possible to assess Nb73::HA:6His [group 3] using SPR due to the inconsistent yield and impure production of this nanobody from bacteria). Sensor chips were coated with purified ${ }_{6 H \mathrm{His.:}}$ TbBILBO1 by amine coupling and probed sequentially at increasing concentrations with purified $\mathrm{Nb9} 9_{\mathrm{HA}: 6 \mathrm{His}}$ and $\mathrm{Nb} 48_{\text {:HA:6His* }}$. The $\mathrm{Nb}$ binding results are shown on the sensorgrams (Fig. $2 \mathrm{H}$ and I), respectively, and are from a titration data set of three concentrations of the nanobodies: $31.3 \mathrm{nM}, 125 \mathrm{nM}$, and $500 \mathrm{nM}$. These results illustrate that $\mathrm{Nb9}_{\text {:HA:6His }}$ and $\mathrm{Nb} 8_{\text {:HA:6His }}$ bind to ${ }_{6 \mathrm{His:}}$ TbBILBO1 but do not behave kinetically in the same way. The superposition of the sensorgrams with the fitting curves validates the Langmuir 1:1 model of interaction for analyzing them. $\mathrm{Nb9}_{: \mathrm{HA}: 6 \mathrm{His}}$ displays higher rate constants than $\mathrm{Nb} 48_{\text {:HA: } 6 \mathrm{His}}$ with $\mathrm{Nb9}$ :HA:6His $k_{a}$ of $6.54 \times 10^{6} \pm 0.27 \times 10^{6} \mathrm{M}^{-1} \mathrm{~s}^{-1}$ and $k_{b}$ of $98.9 \times 10^{-3} \pm 0.09 \times 10^{-3} \mathrm{~s}^{-1}$ and Nb48:HA:6His $k_{a}$ of $1.74 \times 10^{5} \pm 0.16 \times 10^{5} \mathrm{M}^{-1} \mathrm{~s}^{-1}$ and $k_{b}$ of $1.52 \times 10^{-3} \pm 0.02 \times 10^{-3} \mathrm{~s}^{-1}$. Because of a 100 -fold lower rate of dissociation and despite a 10-fold lower rate of association, $\mathrm{Nb} 48_{\text {:HA: } 6 \mathrm{His}}$ displays the highest affinity for ${ }_{6 \text { His: }}$ TbBILBO1 


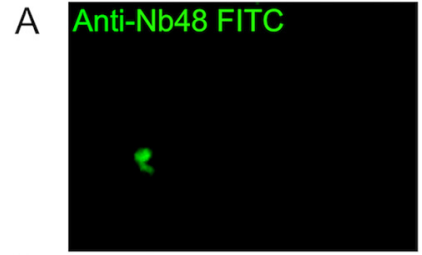

B Anti-Nb48 FITC

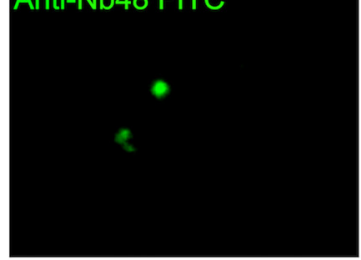

C

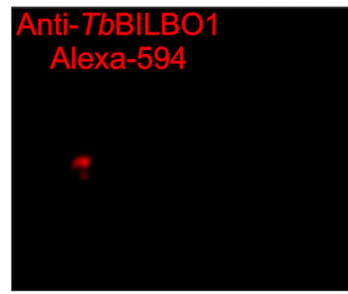

D

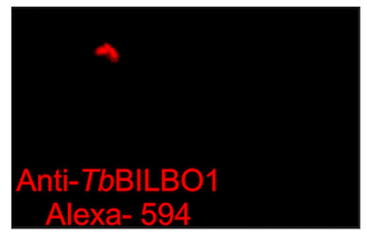

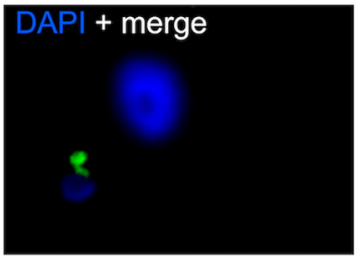
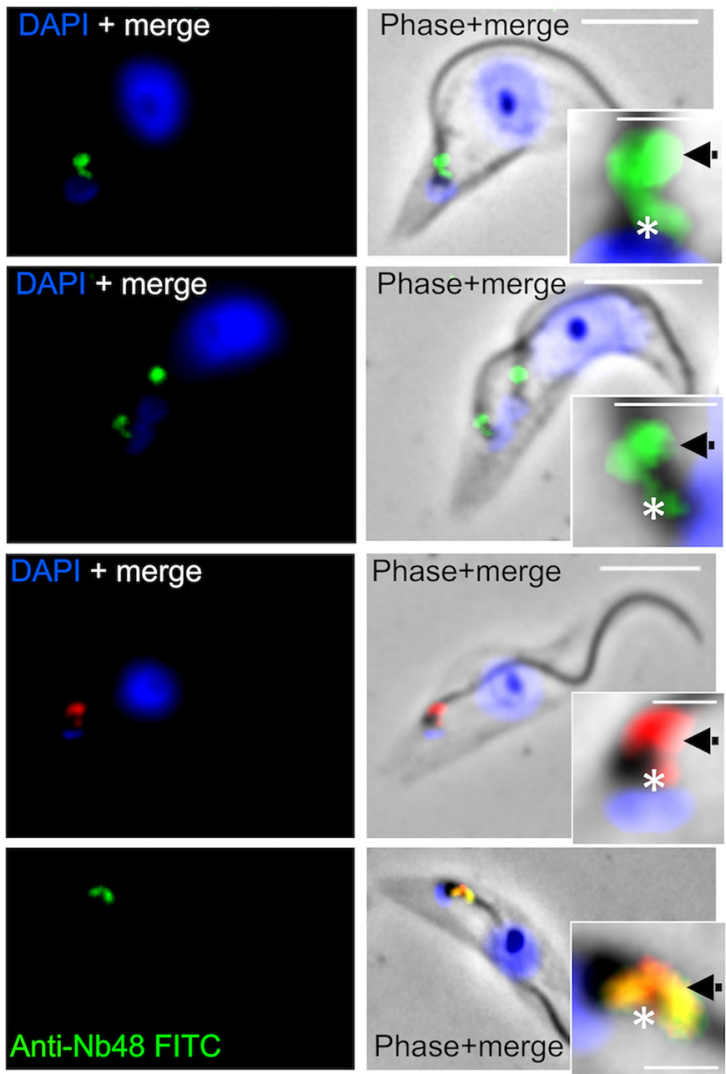

E
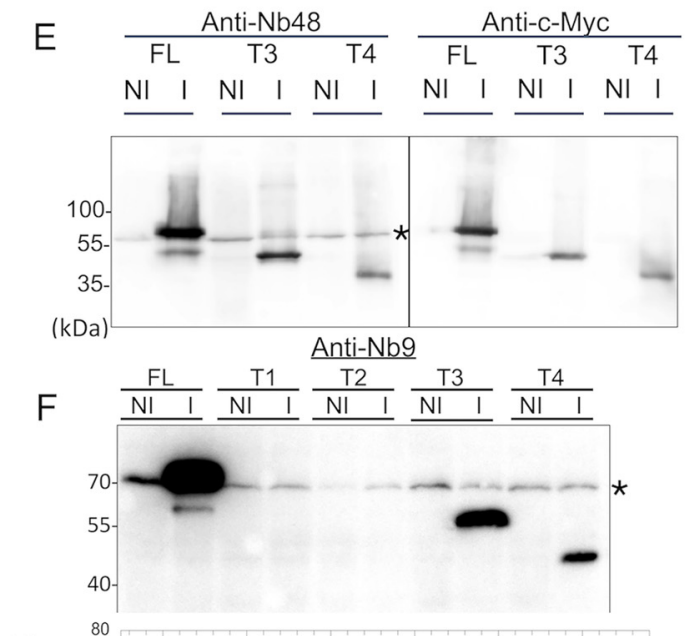

$\mathrm{H}$
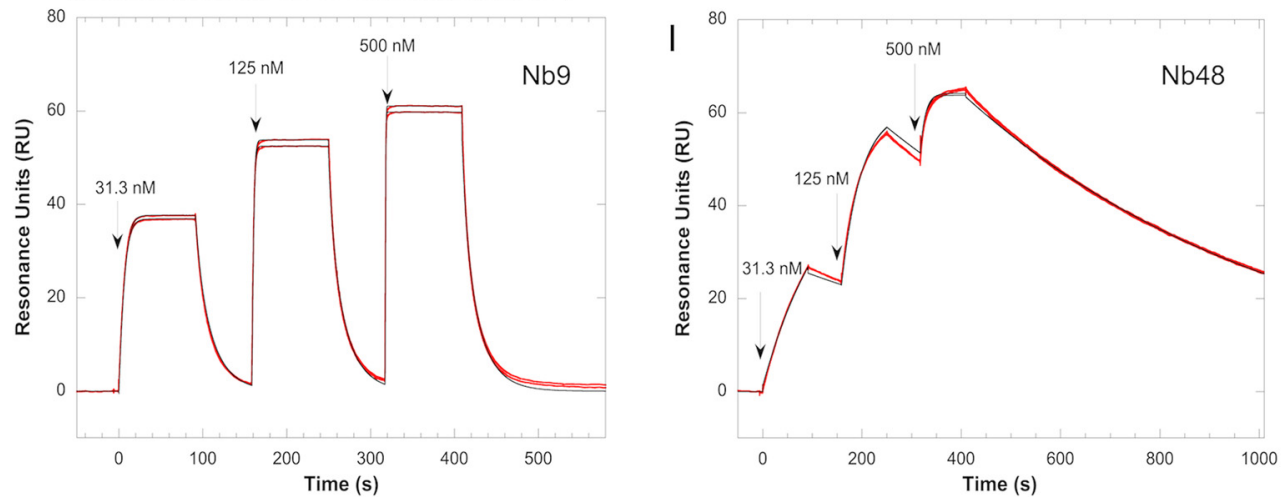

FIG 2 Anti-TbBILBO1 nanobodies Nb48::HA:6His and Nb9::HA:6His bind to TbBILBO1 in vitro. Panels A and B show WT, PCF cytoskeletons probed with $\mathrm{Nb48}:$ HA::6His followed by anti-HA, then FITC-conjugated anti-mouse. (A) A parasite cell in the (Continued on next page) 
with a $K_{D}$ of $8.8 \pm 0.7 \mathrm{nM}$ compared to $\mathrm{Nb}_{\text {:HA: }: 6 \mathrm{His}} K_{D}$ of $15.2 \pm 0.7 \mathrm{nM}$. These values confirm that $\mathrm{Nb} 48_{\text {:HA:6His6 } \times \text { His }}$ has the highest affinity for ${ }_{6 \mathrm{His}:}$ TbBILBO1. Nb48 provided the best results as a tool by IFA, as a probe on Western blotting, and binding to TbBILBO1 as measured by SPR; we therefore decided to focus on Nb48 for further studies.

Anti-TbBILBO1 intrabodies bind to the FPC and the MtQ. Previous studies using stimulated emission depletion microscopy (STED), a technique of switching off the fluorescence of dye molecules by stimulated emission using laser light (49), and immuno-electron microscopy have illustrated that the anti-TbBILBO1 (1-110) rabbit polyclonal antibody labeled both at the FPC and the MtQ. It is important to note here that Esson et al., Albisetti et al., and Dong et al. have shown that TbMORN1, TbSPEF1 (a microtubule binding and bundling protein), and TbSAF1 are localized to the MtQ of flagella, thus clearly indicating that they remain attached to this structure after harsh extraction and indicating that labeling of the MtQ is not artefactual $(22,27,28)$. Given that TbBILBO1 and TbMORN1 are stable on isolated flagella, we questioned whether the FPC could be probed in vivo in trypanosomes by intrananobody (INb) targeting. Consequently, the DNA sequence encoding Nb48 plus a Cterminal 3cMyc tag was cloned into a T. brucei expression vector allowing the tetracycline-inducible expression of the recombinant intrabody 48 (INb48 ${ }_{: 3 \mathrm{cmyc}}$ ). Isolated flagella derived from procyclic cells (PCF) expressing INb48 labeled by probing with anti-cMyc and anti-TbBILBO1 (1-110), showing colocalization of TbBILBO1 and INb48 (Fig. 3A). For convenience, the use of INb48 is displayed in these figures as anti-INB48 rather than indicating the tag used on the intrabody. Immuno-electron microscopy was also carried out on flagella that were double labeled using anti-cMyc and antiTbBILBO1 antibodies (Fig. 3B). Both the IFA and the electron micrographs of these experiments revealed that INb48 ${ }_{: 3 \mathrm{cmyc}}$ colocalizes with anti-TbBILBO1 on the FPC but also on the proximal region of the MtQ as described earlier in the IFA studies shown in Figures $2 A$ to $D$. We also carried out a temporally shorter expression assay of INb48:3cMyc which revealed that it is observed on the FPC and the MtQ at $3 \mathrm{~h}$ postinduction (Fig. S2D). Since INb9 also localized with $\mathrm{TbBILBO} 1$ on the FPC, it was also tested at reduced expression time $(6 \mathrm{~h})$ and was shown on the FPC and colocalized with anti-TbBILBO1 (Fig. S2E).

The expression of anti-TbBILBO1 intrabodies is cytotoxic in T. brucei. Because anti-TbBILBO1 intrabodies colocalize with TbBILBO1 on isolated flagella, we hypothesized that INbs bind tightly to TbBILBO1 and, as a consequence, may disturb FPC formation or function. To test this hypothesis, we monitored the effect of expression of INb48: using different concentrations of tetracycline (Fig. 4A). Tetracycline concentrations of $0.1 \mathrm{ng} / \mathrm{ml}$ and $1 \mathrm{ng} / \mathrm{ml}$ had no effect on cell growth compared to that of the noninduced cells (-Tet; red line). However, concentrations from $5 \mathrm{ng} / \mathrm{ml}$ to $1 \mu \mathrm{g} / \mathrm{ml}$ induced a rapid growth defect from $24 \mathrm{~h}$ postinduction (hpi) and cell death after $24 \mathrm{hpi}$. The change in growth rates related to different doses of tetracycline indicates that the lethality of INb48::3cMyc expression in PCF is dose dependent. Considering these results, we chose a tetracycline

FIG 2 Legend (Continued)

$1 \mathrm{~K} 1 \mathrm{~N}$ stage of the cell cycle. (B) A cell in the $2 \mathrm{~K} 1 \mathrm{~N}$ stage. Both cell stages show Nb48::HA:6His labeling on the FPC (black arrow) and MtQ (white asterisk). The MtQ signal was not always apparent on every cell imaged, which could be due to modulations between cell cycle stages. (C) WT, T. brucei cytoskeleton probed with anti-TbBILBO1 (1-110) followed by anti-rabbit Alexa fluor 594. (D) WT cytoskeleton probed with anti-TbBILBO1 and Nb48 :HA:6His showing complete colocalization. (E) Western blotting of whole-cell extracts of $T$. brucei PCF cells expressing TbBILBO1 full-length (FL) and TbBILBO1 truncations, probed with $\mathrm{Nb} 48_{\mathrm{HA}: 6 \mathrm{His}}$. Noninduced ( $\left.\mathrm{NI}\right)$ and induced (I) trypanosomes expressing TbBILBO1 $1_{: 3 \mathrm{cMyc}} \mathrm{FL}$

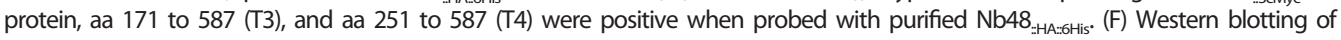
whole-cell extracts of $T$. brucei PCF cells expressing TbBILBO1 full-length (FL) and TbBILBO1 truncations probed with Nb9. Noninduced (NI) and induced (I) trypanosomes expressing TbBILBO1 $1_{: 3 \mathrm{cMyc}}$ FL protein, aa 171 to 587 (T3), and aa 251 to 587 (T4) were positive when probed with purified Nb9. ${ }_{\text {HA-6His. }}$ Note: all proteins are running slightly faster than predicted size; FL should run at $70 \mathrm{kDa}, \mathrm{T} 3$ should run at $49 \mathrm{kDa}$, and T4 should run at $40 \mathrm{kDa}$. The black asterisk indicates labeling of endogenous TbBILBO1 seen across all samples. (G) A schematic diagram of cMyc-tagged TbBILBO1 full-length (FL) and truncations. Note that $\mathrm{T} 1$ and $\mathrm{T} 2$ are soluble. $\mathrm{Nb} 9_{\mathrm{HA}: 6 \mathrm{His}}$ and $\mathrm{Nb} 48_{\text {:HA:6His }}$ bind to a region within aa 251 and 587 of TbBILBO1. (H) Kinetic analysis by

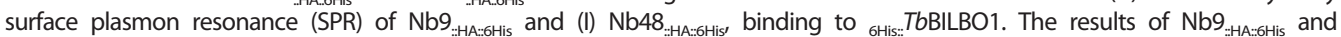
$\mathrm{Nb} 8_{\mathrm{HA}: 6 \mathrm{His}}$ binding are shown on the sensorgrams, respectively, and are represented by the red curves. The black lines represent the theoretical fit of each $\mathrm{Nb}$ as obtained from the Biaevaluation software to a kinetic titration data set of three concentrations of the nanobodies in vitro. Nb9 $9_{\text {:HA:6His }}$ displays a good affinity for ${ }_{6 \mathrm{His:}}$ TbBILBO1 with a $K_{D}$ of $15.2 \pm 0.7 \mathrm{nM}$; however, $\mathrm{Nb} 48_{\mathrm{:HA}: 6 \mathrm{His}}$ displays the highest affinity for ${ }_{6 \mathrm{His:}} \mathrm{TbBILBO} 1$ with a $K_{D}$ of $8.8 \pm 0.7 \mathrm{nM}$. Scale bar is $5 \mu \mathrm{m}$, inset is $1 \mu \mathrm{m}$. 
Alexa 594

A

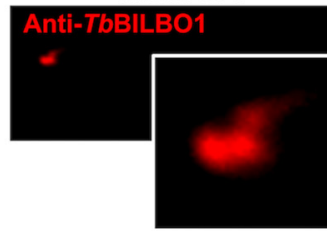

FITC

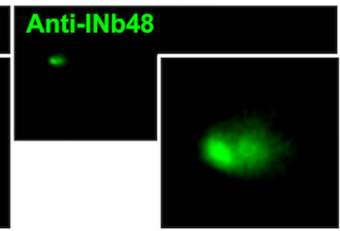

Merge

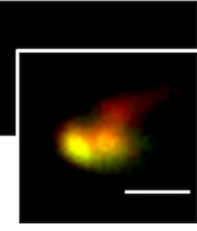

Phase

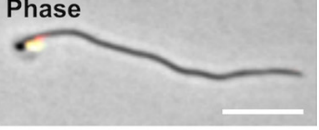

B

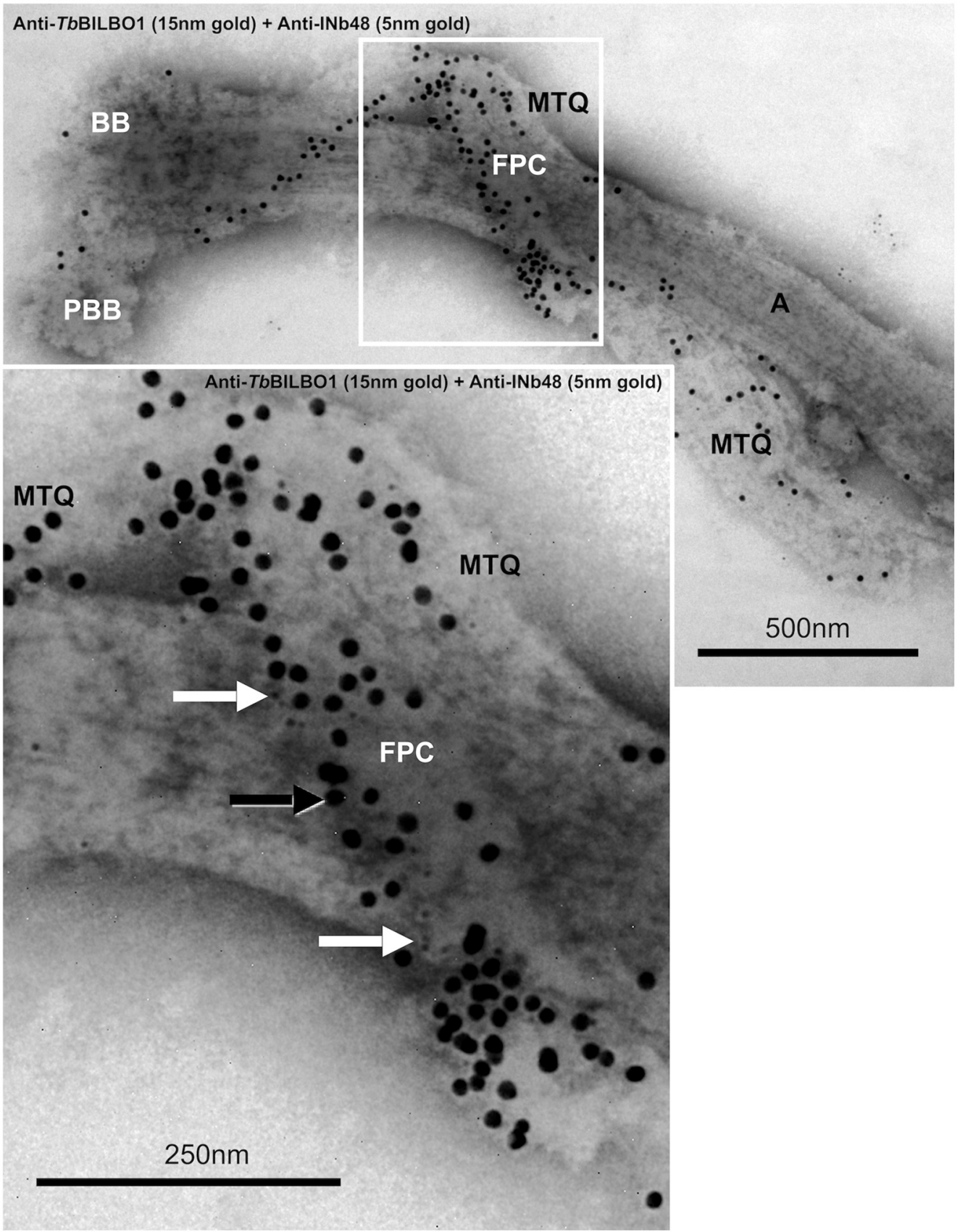

Anti-INb48 $(5 \mathrm{~nm}$ gold beads $=$ white arrow $)$

Anti-TbBILBO1 (15nm gold beads = black arrow)

FIG 3 INb48 $:$ :зсмус binds to TbBILBO1 in vivo. (A) IFA colocalization of INb48::3смус with TbBILBO1 on isolated T. brucei PCF flagella. Scale bar of $5 \mu \mathrm{m}$, inset of $1 \mu \mathrm{m}$. (B) Immuno-electron micrographs of a purified flagellum from a trypanosome expressing INb48. TbBILBO1 (15 nm gold beads, black arrow). Both $5 \mathrm{~nm}$ and $15 \mathrm{~nm}$ gold beads are also clearly visible on the MtQ. BB, basal body; PBB, probasal body; FPC, flagellar pocket collar; MtQ, microtubule quartet; $\mathrm{A}$, axoneme. Scale bar is $500 \mathrm{~nm}$, inset is $250 \mathrm{~nm}$. 
A

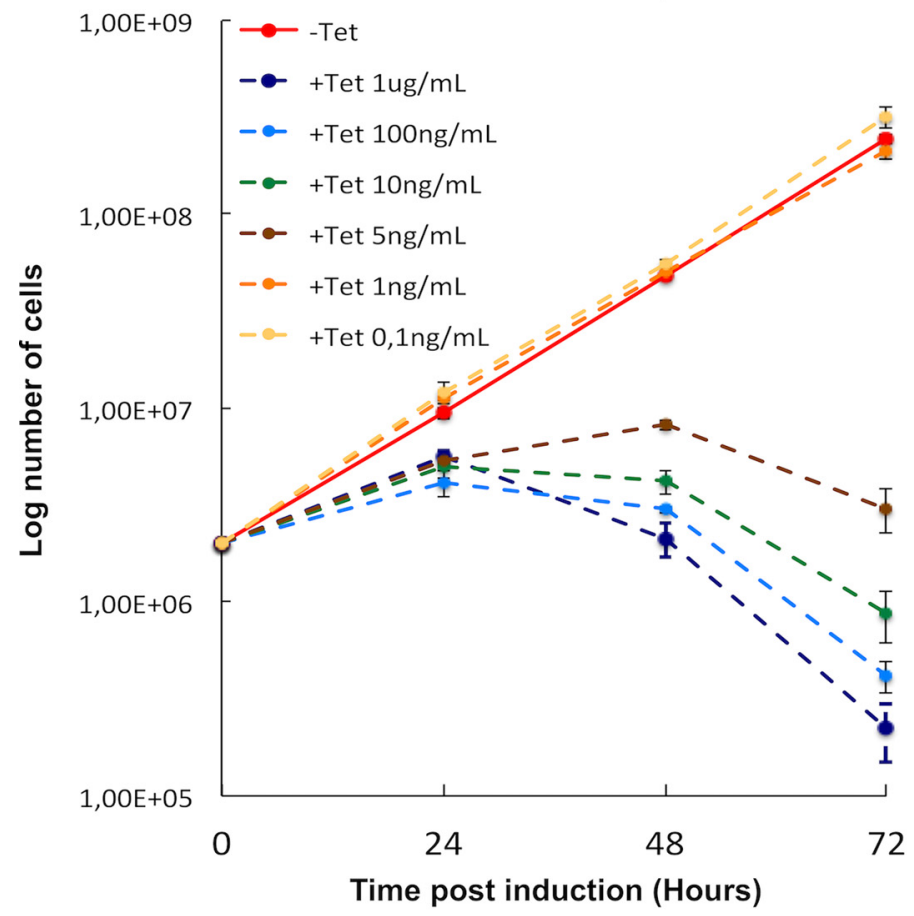

$\mathrm{E}$

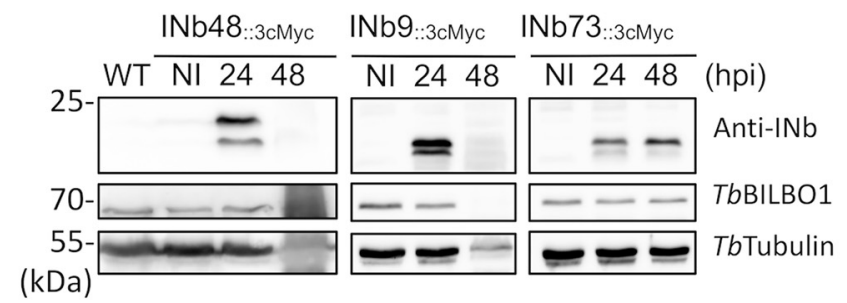

B

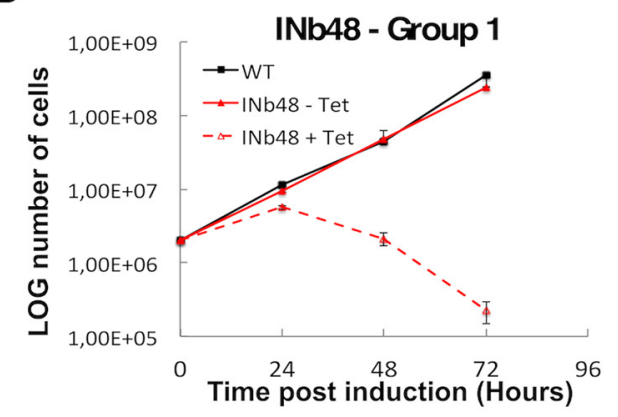

C

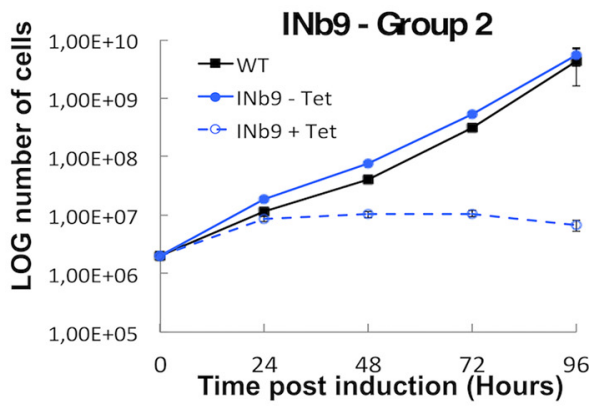

D

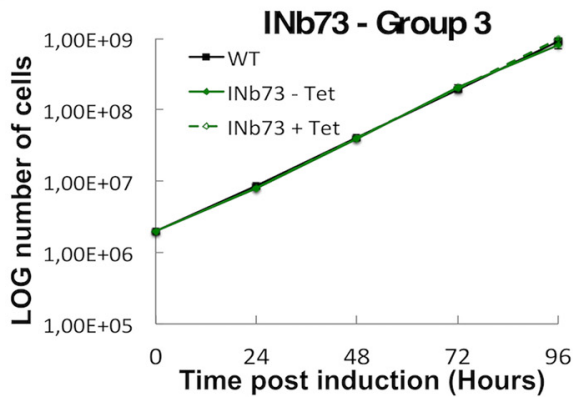

FIG 4 INb48: $:$ ссмус expression is trypanocidal. (A) Growth curves of trypanosome cells expressing INb48 ${ }_{: 3 c M y c}$ Tetracycline dose-dependent effect on trypanosome growth and cytotoxicity for INb48:

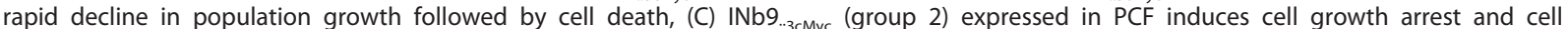
death, and (D) INb73::3смyc (group 3) expressed in PCF has no effect on population growth. (E) Western blot of INb48 ::3cMyc' INb9::3cMyc and INb73.:3cMyc expression in PCF showing expression only in induced cells. Rapid degradation of protein due to cell death at 48 hpi is observed for $I N b 48_{: 3 c \text { Myc }}$ and $I N b 9_{: 3 c M y c}$. Time postinduction is in hours.

concentration of $1 \mu \mathrm{g} / \mathrm{ml}$ as the standard concentration for INb48: ${ }_{\text {:3cMyc }}$ INb9::3cMyc and INb73:3cMyc induction. After induction of the expression of these three INbs in trypanosomes, daily samples were taken for cell counts (Fig. 4B to D) and Western blotting (Fig. 4E). Independent induction of each INb resulted in phenotypes that ranged from rapid cell death from $24 \mathrm{hpi}$ for INb48:3cмyc (Fig. 4B) to a reduction in cell growth rate for INb9: ${ }_{: 3 \mathrm{cmyc}}$ (Fig. 4C) or no change in growth rate for INb73:зсмус (Fig. 4D). Western blotting of $\mathrm{INb}$ expression is shown in Figure $4 \mathrm{E}$ and illustrates robust expression of all INbs at $24 \mathrm{~h}$ postinduction in trypanosomes. After $48 \mathrm{hpi}$ of intrabody expression, the signals for $1 \mathrm{Nb} 48_{: 3 \mathrm{scMyc}}$ and INb9: ${ }_{: 3 \mathrm{cmyc}}$ were lost and the signal for TbBILBO1 and tubulin control proteins showed signs of degradation, indicating some degree of cell death in the population. However, no degradation was observed in cells expressing $1 \mathrm{Nb} 73_{\text {:3cMyc }}$ which, importantly, showed no effect on cell growth. As a negative control, an intrabody against green fluorescent protein (INbGFP::3cMyc) was expressed in trypanosomes, and as expected, no cell growth defect or phenotype was observed despite its strong expression and cytoplasmic localization (Fig. S3A to C). These data demonstrate that expression of INb48: $: 3 c \mathrm{Myc}$ and INb9::3cMyc are 
lethal, with INb48::3cMyc showing the strongest effect, while expression of INb73::3cмyc had no effect on cell growth. The data also suggest that INb48 :3смус $_{\text {and INb9: }: 3 \mathrm{cMyc}}$ bind to TbBILBO1 in vivo and somehow hinder its function.

Intrabody 48 (INb48::3cMyc) binds to TbBILBO1 and induces TbBILBO1 RNAi knockdown phenotypes. Trypanosome cell cycle stages can be defined based on the arrangement and number of nuclei and mitochondrial genome (kinetoplasts) present in the cell, and wild-type cells should never possess more than the 2K2N DNA complement (50). RNAi knockdown of TbBILBO1 in cultured PCF cells results in cell cycle arrest at the $2 \mathrm{~K} 2 \mathrm{~N}$ stage, producing cells with elongated posterior ends, new flagella that were detached from the length of the cell body, and inhibition of new FP formation (13). Figure 5A shows noninduced (-Tet, NI), whereby the $\mathrm{NI}$ trypanosomes display the classic immunofluorescence signal for TbBILBO1 with the annular labeling at the FPC and along the MtQ between the FPC and the basal bodies (BB). After $24 \mathrm{hpi}$ of INb48: morphological phenotypes were observed where the new flagellum was detached from the length of the cell body and cells demonstrated elongated posterior ends. In TbBILBO1 RNAi knockdown experiments, very little BILBO1 protein was observed at the new flagellum and only low levels were observed at the old flagella. However, when INb48::3cMyc was expressed, both INb48: $: 3$ мyс and TbBILBO1 were sometimes observed at the old FPC and also at the base of the detached new flagellum (Fig. 5B, asterisk). More importantly, rather than the classic annular and MtQ signals observed after anti-TbBILBO1 labeling, we observed both TbBILBO1and INb48: $: 3$ Myc signals extending along the cell (Fig. 5B, merged inset). This result demonstrates that no new FPC was formed after INb48 TbBILBO1 RNAi-like phenotype and, importantly, that INb48 ${ }_{\text {:3cMyc }}$ binding to TbBILBO1 disturbs FPC formation.

We decided to investigate the organization of the hook complex $(\mathrm{HC})$, a structure intimately associated with the FPC, by immunolabelling of TbMORN1 (28). The HC is an essential structure positioned distal to the FPC, forming a distinctive hook shape, and is considered to be indirectly involved in the regulation of entry of molecules into the flagellar pocket. Figure $5 \mathrm{C}$ illustrates the normal location and hook-like shape of antiTbMORN1 labeling in noninduced cells. However, at 24 hpi of INb48::3cMyc expression, TbMORN1 labeling was elongated distally along the flagellum, and the hook shape was lost (Fig. 5D). Importantly, little or weak TbMORN1 labeling is observed at the base of the new flagellum, suggesting that $\mathrm{HC}$ biogenesis is also strongly affected in cells expressing $\mathrm{INb} 48_{: 3 \mathrm{scMyc}} \mathrm{A}$ consistent observation in cells expressing INb48 detached from the length of the cell body, i.e., only attached at the base, an abnormal phenotype strongly resembling the TbBILBO1 RNAi knockdown phenotype (13). We further characterized the detached new flagellum phenotype by colabeling with anti-TbPFR2 antibody (51). TbPFR2 is a protein of the paraflagellar rod (PFR) that is present alongside the axoneme of the flagellum of wild-type T. brucei (51) as shown in noninduced cells (Fig. 5E). In induced cells (24 hpi), TbPFR2 labeling was observed along the old flagellum and the newly detached new flagellum (Fig. 5F). In this cell, INb48:3cMyc was observed only at the old FPC, indicating that a new flagellum and PFR was formed in the absence of the FPC. To investigate any effect of INb48 ${ }_{: 3 \text { см Mc }}$ expression on the basal bodies (BB), an antibody marker anti-TbBLD10 was used, which was raised against TbBLD10, an essential protein in probasal body biogenesis (52). Figure $5 \mathrm{G}$ shows a noninduced $T$. brucei cell with the typical labeling of the mature and probasal bodies. At 24 hpi of INb48 ${ }_{: 3 c \mathrm{Myc}}$ the labeling of both the pro- and mature basal bodies of the mother and daughter flagella was unaffected (Fig. $5 \mathrm{H}$ ). We also checked a longer INb48::зсмус expression (48 hpi) by IFA and demonstrated that cells were severely compromised. Cells showed cell cycle disruption, including the presence of multiple flagella (Fig. S3D and E). Also, the TbBILBO1 labeling revealed extended structures demonstrating that the shape of the polymers formed by TbBILBO1 were strongly affected and were unable to form the typical annular FPC.

The phenotypes induced after INb48: more detail by transmission electron microscopy (TEM). TEM micrographs of thin sections through the FP $\left(^{*}\right)$ of a wild-type cell (Fig. $6 \mathrm{~A}$ ) illustrate that the flagellum passes through this structure and that its transition zone is positioned within the FP lumen (black arrow). 
FITC
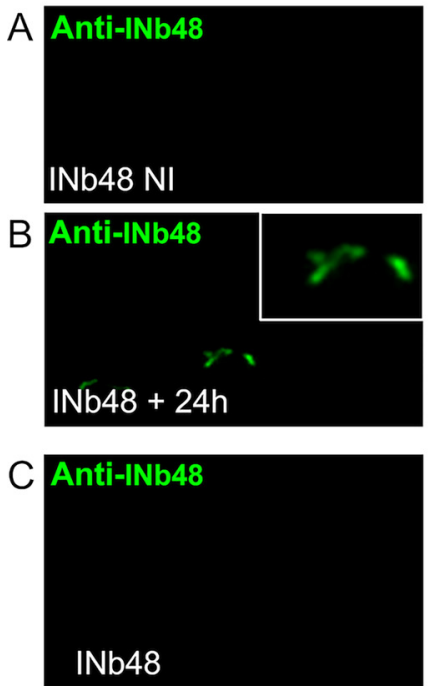

D Anti-INb48
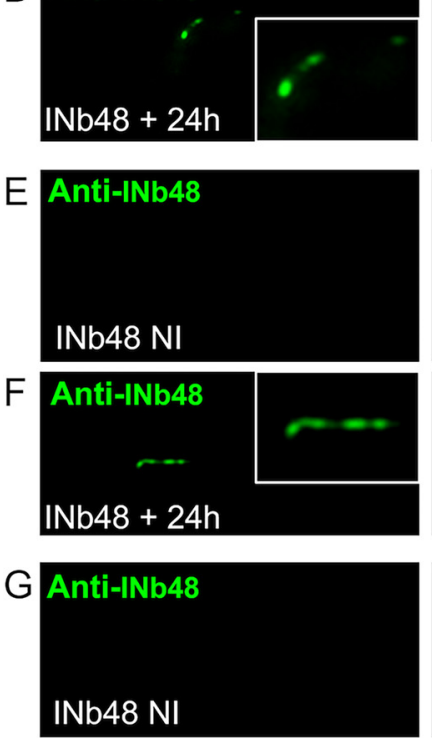

H Anti-INb48 =

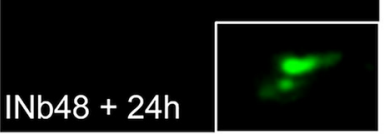

Alexa- 594
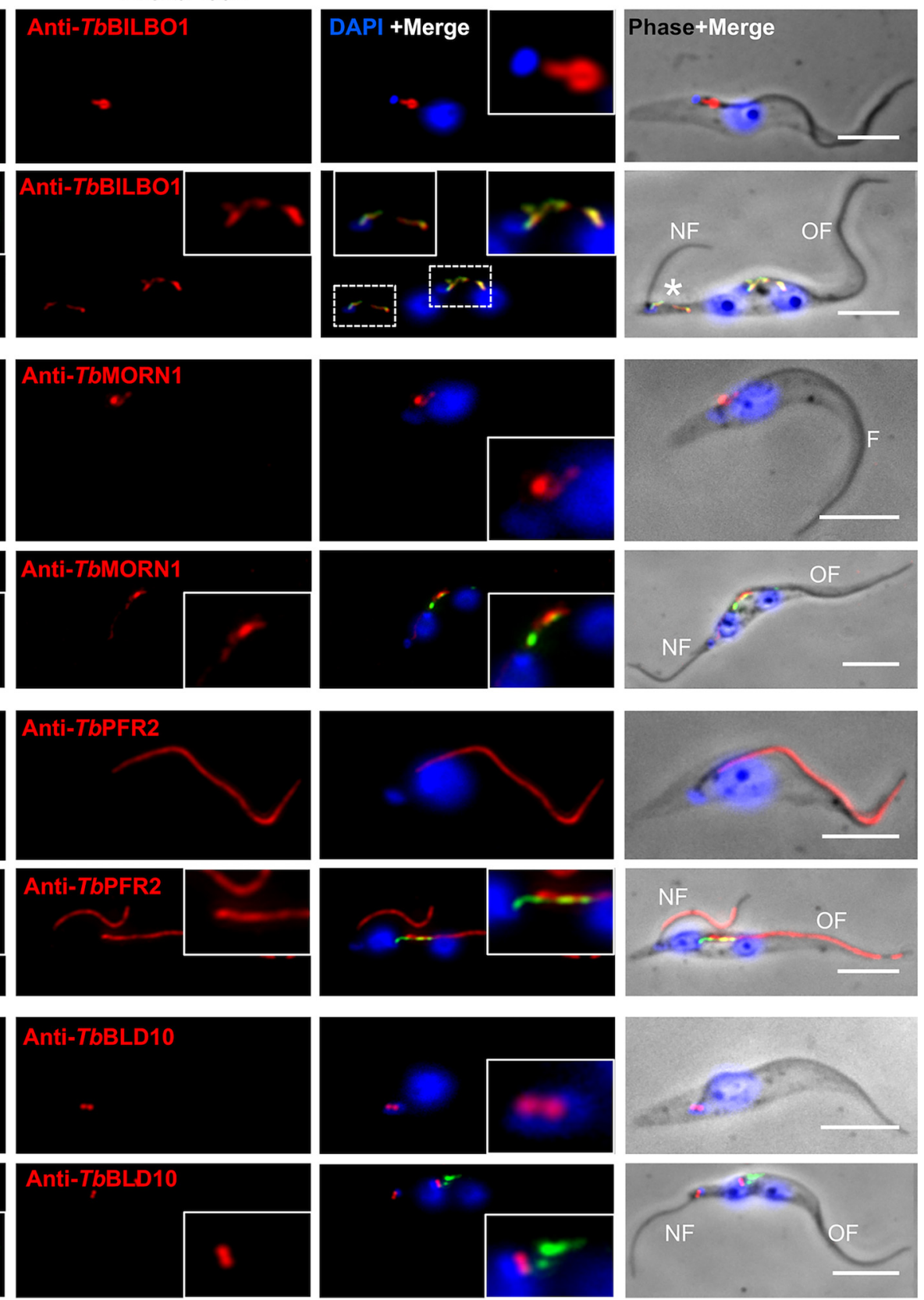

FIG 5 INb48 cells probed with anti-cMyc as a probe for INb48 $: 3$ смус (green) and anti-TbBILBO1 (red), indicating the absence of INb48 $: 3 c \mathrm{cmyc}$ labeling in noninduced cells. (B) An INb48..3cMyc-induced (Ind) T. brucei cell at 24 hpi showing colocalization of INb48..3cmyc (green) with a disorganized signal for TbBILBO1 (linear polymer formation instead of the typical annular shape) and a posteriorly localized, detached new flagellum (labeled as NF). Asterisk (*) denotes the presence of colocalization of INb48. (labeled as OF) and at the base of the new detached flagellum (labeled NF). (C) Noninduced (NI) trypanosome showing correct location of TbMORN1 labeling (red) at the base of the flagellum (labeled as F), with the classic hook and shank composition; there is no anti-cMyc signal in this $\mathrm{NI}$ cell. (D) At $24 \mathrm{hpi}$ (Ind), INb48 along the axoneme; TbMORN1 labeling has changed from the classic hook shape and is seen as an extended linear form extending distally from the FPC region along the direction of the old flagellum (OF). (E) Noninduced (NI) trypanosome showing typical labeling of TbPFR2 (red) along the flagellum. (F) $24 \mathrm{hpi}$ Ind INb48: ${ }_{\text {: смус }}$ (green) showing a trypanosome cell with both the old (OF) and new flagella (NF) labeled with TbPFR2. INb48::3смyс labeling is observed only at the old FPC extending along anteriorly in this cell. (G) A noninduced (NI) trypanosome cell probed with anti-TbBLD10 (red), labeling both the mature and probasal bodies (BB) at the base of the flagellum. $(\mathrm{H}) \mathrm{At} 24 \mathrm{hpi}(+\mathrm{Tet})$ of INb48::зсмус (green), a detached new flagellum (NF) is seen at the tip of at an extended posterior end; anti-TbBLD10 labeling is seen associated with both the new and old flagella (red); INb48::3cMyc labeling is seen mainly in the region of the FPC of the old flagellum (green). Scale bar is $5 \mu \mathrm{m}$. 

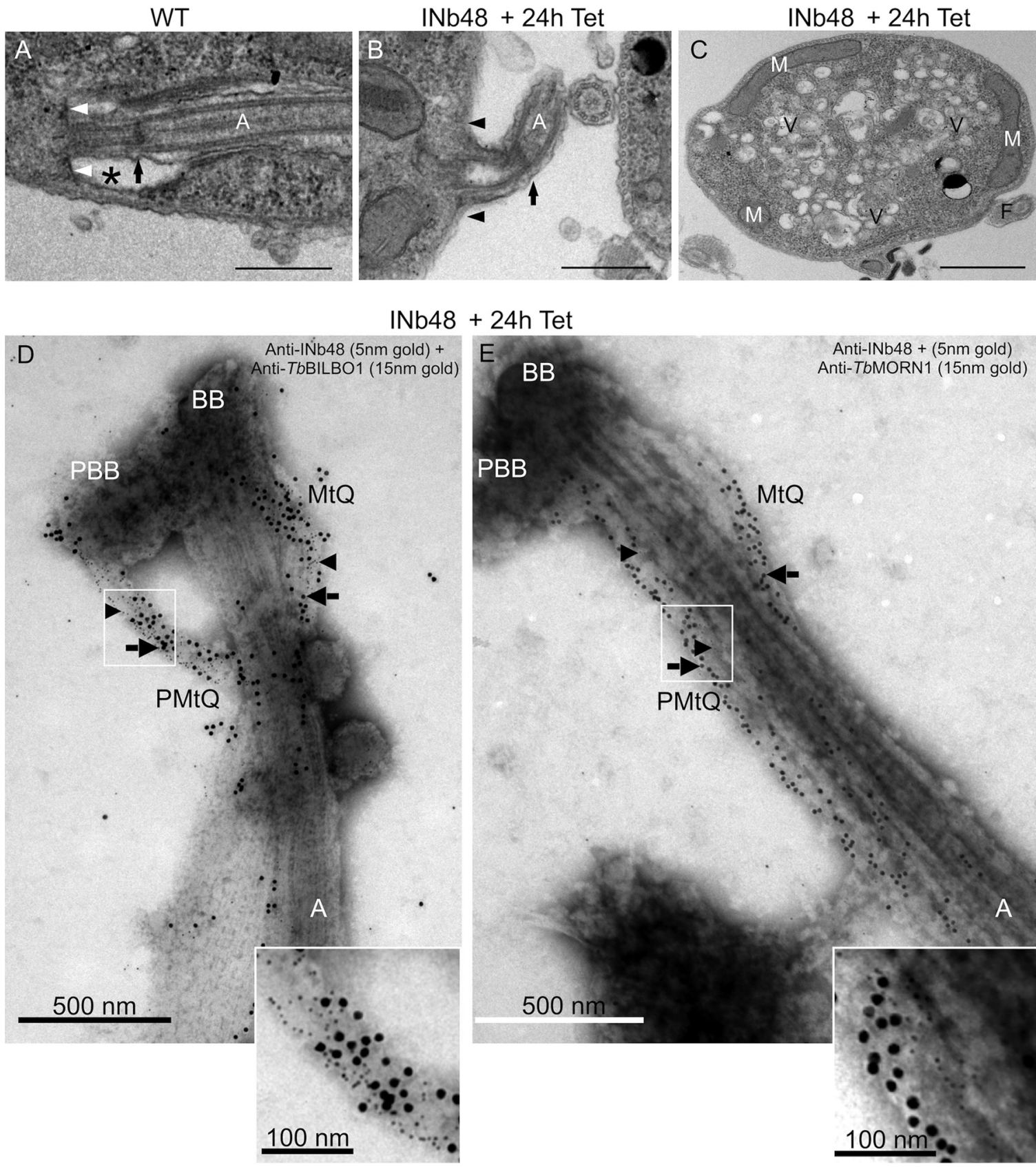

Anti-INb48 $(5 \mathrm{~nm}$ gold beads $=$ black arrowheads $)$ Anti-TbBILBO1 1-110 (15nm gold beads = black arrows)

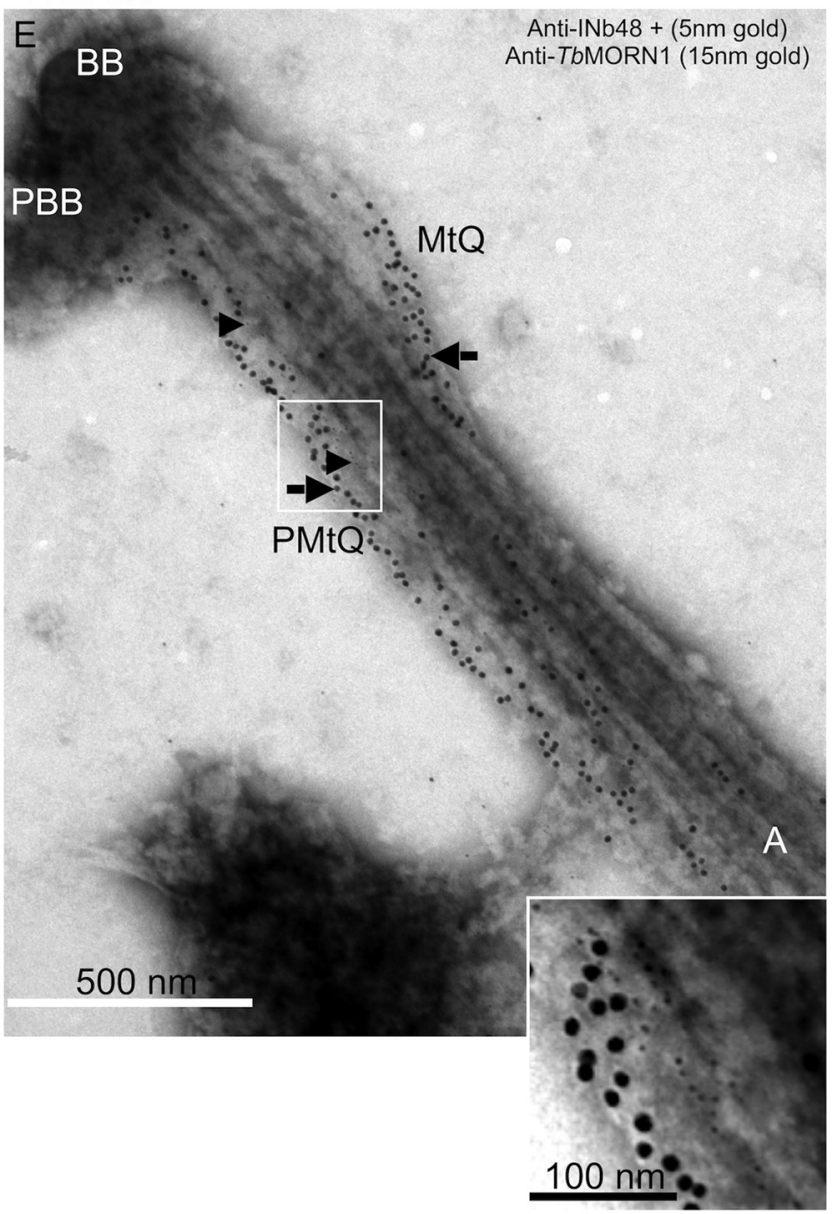

Anti-INb48 $(5 \mathrm{~nm}$ gold beads $=$ black arrowheads $)$ Anti-TbMORN1 $(15 \mathrm{~nm}$ gold beads = black arrows $)$

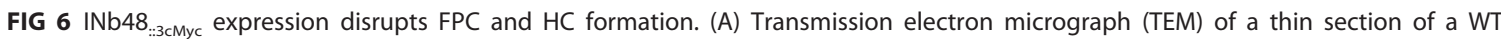
PCF T. brucei cell showing the typical flagellar pocket (FP) (*), the base of the flagellar pocket (white arrows), flagellar axoneme (A), and exit site of the axoneme from the FP membrane; the transition zone is indicated by a black arrow within the FP. (B) Illustrates a detached flagellum from a thin section of PCF cells expressing INb48::3cмyс $24 \mathrm{hpi}$ (+Tet). The FP has not been formed and microtubules are present where the FP membrane should be located (black arrowheads). The transition zone of the axoneme is outside the cell body (black arrow). (C) TEM of a thin section of a cell expressing INb48 ${ }_{: 3 c M y c}$ for $24 \mathrm{hpi}$ (+Tet), showing accumulation of intracellular vesicles. $\mathrm{M}$, mitochondrion; $\mathrm{Vm}$, vesicles; F, flagellum. Scale bar is $1 \mu \mathrm{m}$. (D) Immuno-electron micrograph (iEM) of isolated flagella of PCF T. brucei after $24 \mathrm{hpi}(+\mathrm{Tet})$ of INb48::3cMyc expression. INb48 as black arrowheads) colocalizing with anti-TbBILBO1 (15 nm gold beads represented as black arrows) on the MtQ between the BB and the expected location of the FPC; note that the FPC is absent. (E) IEM of isolated flagella of PCF T. brucei after $24 \mathrm{hpi}(+$ Tet) of INb48. elongated labeling of TbMORN1 (15 $\mathrm{nm}$ gold beads represented by black arrows). Note that in trypanosome cells expressing INb48: :смус $_{\text {c }}$ the new FPC is absent and the Hook Complex that is normally represented by TbMORN1 has lost its hook shape. BB, basal body; PBB, probasal body; MtQ, microtubule quartet; A, axoneme; PMtQ, presumed probasal body MtQ. Scale bar is $500 \mathrm{~nm}$, inset scale bar is $100 \mathrm{~nm}$. 
INb48 absence of an FP (Fig. 6B, black arrowheads indicate the position where the FP should be formed), and the transition zone is now external to the cell (black arrows). These cells also accumulated cytoplasmic vesicles (Fig. 6C). These abnormal features are typical of TbBILBO1 RNAi knockdown phenotypes (13) indicating that TbBILBO1 function was indeed hampered by the expression of INb48: $24 \mathrm{hpi} \mathrm{(+Tet)} \mathrm{and} \mathrm{probed} \mathrm{them} \mathrm{with} \mathrm{anti-cMyc} \mathrm{(for} \mathrm{INb48:}{ }_{\text {:cMyc }}$ ) and anti-TbBILBO1 or, in a separate experiment, anti-cMyc and anti-TbMORN1. The flagella were prepared for negative staining and visualized by transmission electron microscopy (TEM). The colabeling of INb48 MtQ (Fig. 6D and E). TbBILBO1 was located on the MtQ proximal to the basal bodies (Fig. 6D), whereas TbMORN1 was primarily on the MtQ distal to the basal bodies (Fig. 6E). Interestingly, however, the typical annular FPC structure was absent in INb48 trypanosomes, explaining the detached flagellum phenotype. In Figure 6D, INb48: (arrowheads) is present alongside TbBILBO1 (arrows) extending from the basal bodies on the MtQ to the location where the FPC should be present. Figure 6 E shows TbMORN1 labeling and shows in more detail that in the presence of INb48::3смус (arrowheads), it was observed on the MtQ (arrows) extending in a linear fashion proximally along the flagellum but not distally. These data demonstrate that the FPC and HC are structurally perturbed by the expression of $\mathrm{INb} 48_{: 3 \mathrm{cmyc}}$ in T. brucei.

Expression of INb48::3cMyc disrupts the cell cycle in PCF T. brucei. The organization of kinetoplasts and nuclei in INb48::3смyc-expressing cells was analyzed at different time points (Fig. 7A). The percentage of noninduced (-Tet) cell cycle stages were $74.4 \%$ in the $1 \mathrm{~K} 1 \mathrm{~N}$ stage (blue bars), $13.9 \%$ in the $2 \mathrm{~K} 1 \mathrm{~N}$ stage, and $9.4 \%$ in the $2 \mathrm{~K} 2 \mathrm{~N}$ as published previously (13). A change was observed in trypanosomes expressing INb48::зсмус for $12 \mathrm{~h}(+$ Tet $12 \mathrm{~h}$ ), with a reduction in $1 \mathrm{~K} 1 \mathrm{~N}$ cells and an increase in $2 \mathrm{~K} 1 \mathrm{~N}$ and $2 \mathrm{~K} 2 \mathrm{~N}$ cells. There was also the appearance of abnormal $\mathrm{K} / \mathrm{N}$ phenotypes, such as $1 \mathrm{~K} 2 \mathrm{~N}$ $(1.5 \%)$, when the nucleus had divided before the kinetoplast and cells with other abnormal $\mathrm{K} / \mathrm{N}$ ratios, including cells with more than $2 \mathrm{~K}$ and/or $2 \mathrm{~N}$, annotated XKXN (1.6\%). As mentioned previously, when INb48::3смyc was expressed in trypanosome cells, they exhibited detached flagella (18.3\%). After $24 \mathrm{hpi}$ (+Tet $24 \mathrm{~h}$ ), this profile continued to augment with a further reduction in $1 \mathrm{~K} 1 \mathrm{~N}(44.20 \%)$ and a further increase in $2 \mathrm{~K} 2 \mathrm{~N}$ cells $(27.5 \%)$. Additionally, the number of $1 \mathrm{~K} 2 \mathrm{~N}$ (abnormal) cells increased to $4.3 \%$, and $5.9 \%$ of the population showed XKXN phenotype. Finally, the number of cells with detached flagella had also increased to $35.4 \%$. INb48::3cMyc-expressing cells in the $2 \mathrm{~K} 2 \mathrm{~N}$ stage were further investigated and categorized according to linear organization of $\mathrm{K}$ and $\mathrm{N}$ from posterior to anterior orientation (Fig. 7B). For noninduced cells (-Tet), most 2K2N trypanosomes had the normal KNKN organization with both flagella attached along the cell body (green bar). At $12 \mathrm{hpi}$, the number of normal $2 \mathrm{~K} 2 \mathrm{~N}$ population had decreased (from 93.5 to $45.8 \%$ ) with the appearance of RNAi TbBILBO1 knockdown-like $2 \mathrm{~K} 2 \mathrm{~N}$ cells. A total of $36.44 \%$ of the + Tet $12 \mathrm{~h}$ total $2 \mathrm{~K} 2 \mathrm{~N}$ population had detached flagella and an extended posterior end, which also included $15.8 \%$ with a K_NKN organization (here the underscore refers to an extended end, orange bar). A total of $13.1 \%$ of the abnormal population had a K_KNN kinetoplast organization (red bar), and $7.6 \%$ had a poorly segregated nuclei $\mathrm{K} \_N \mathrm{NNN}^{*}$ (yellow bar). The other $2 \mathrm{~K} 2 \mathrm{~N}$ population $17.7 \%$ (blue bar) included cells that appeared normal in kinetoplast and nuclear organization KNKN but with detached flagella and cells with oddly misplaced kinetoplast (KKNN) with detached flagella. At $24 \mathrm{hpi}$ (+Tet $24 \mathrm{~h}$ ), the proportion of $2 \mathrm{~K} 2 \mathrm{~N}$ "normal-like" population was significantly reduced to $20.3 \%$ at the expense of abnormal cells mentioned above. The rest of the abnormal cells possessed a detached new flagellum which was distributed over a number of arrangements of $\mathrm{K}$ and $\mathrm{N}$ plus an extended posterior end K_KNN (red bar), K_NKN (orange bar), and K_NKN* (yellow bar) or without extended posterior ends (blue bar). These abnormal phenotypes are a clear indication of disruption of the cell cycle. As with RNAi knockdown of TbBILBO1, it appears that there is a general cell cycle arrest at the $2 \mathrm{~K} 2 \mathrm{~N}$ stage, indicating that the 
A Cell cycle stage after INb48 expression

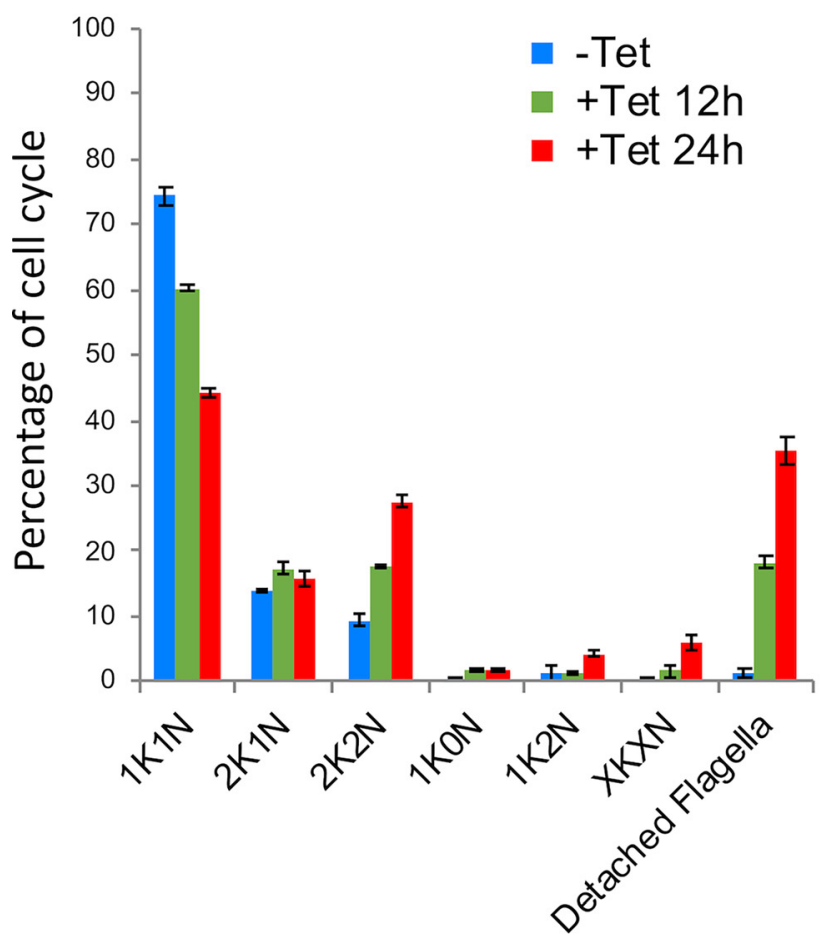

B $\quad 2 \mathrm{~K} 2 \mathrm{~N}$ cell cycle types after INb48 expression

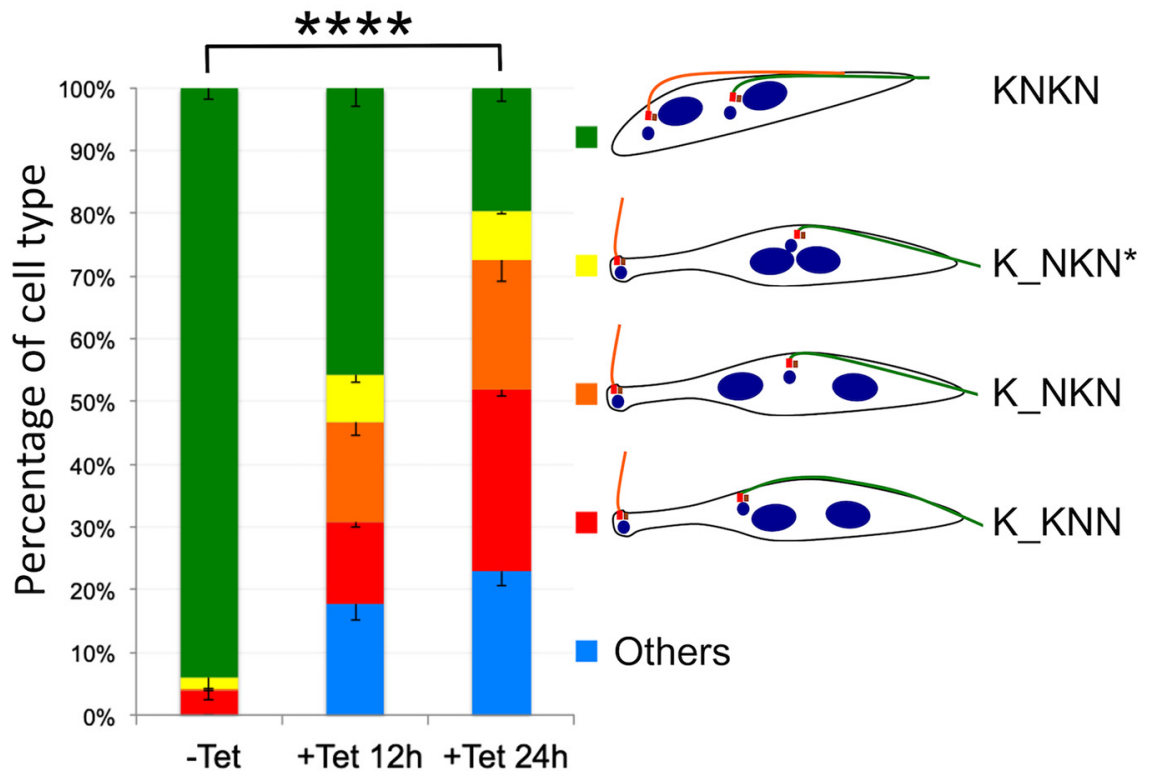

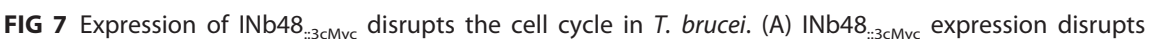
cytokinesis in T. brucei. Bar graph to show the percentage of trypanosomes counted at each stage of the cell cycle for noninduced (-Tet, blue bars), 12 hpi (+Tet 12 h, orange bars), and 24 hpi (+Tet 24 h, red bars) for $1 \mathrm{Nb} 48_{:: 3 \mathrm{cmyc}}$ expression. Note that at $24 \mathrm{hpi}$, approximately $35 \%$ of cells have detached flagella. $1 \mathrm{Nb} 48_{:: 3 \text { смус }}$ expression leads to a decrease in $1 \mathrm{~K} 1 \mathrm{~N}$ and an increase in $2 \mathrm{~K} 1 \mathrm{~N}, 2 \mathrm{~K} 2 \mathrm{~N}$, abnormal $1 \mathrm{~K} 2 \mathrm{~N}$, and XKXN phenotypes. $N$ is equal to 300 trypanosomes per time point, and each time point has been completed in triplicate. (B) Distribution of $2 \mathrm{~K} 2 \mathrm{~N}$ cells after INb48 $: 3$ смус expression in T. brucei showing strong similarities to TbBILBO1 RNAi knockdown. The $2 \mathrm{~K} 2 \mathrm{~N}$ cells were subdivided into 5 categories according to the visual appearance of the cell, i.e., possessing a detached flagellum, an extended posterior end, and abnormal positioning of the kinetoplast and nucleus. ${ }^{* * *}, P<0.0001$. 
cells cannot undergo division. With regard to these cell cycle counts, similar findings were observed with RNAi knockdown of TbBILBO1, suggesting that the crucial steps and structures required for cytokinesis were impaired after INb48: strating that INb48 targeting of TbBILBO1 can be as effective as RNAi knockdown.

Heterologous expression of TbBILBO1 and INb48. Expression of full-length and truncated forms of TbBILBO1 in a U-2 OS heterologous system has been published previously (20). That work demonstrated that expression of full-length TbBILBO1 in mammalian cells induces the formation of linear polymers with comma- and globular-shaped termini. To determine if $\mathrm{Nb} 48$ can bind to TbBILBO1 as an antibody probe in this heterologous system, we expressed full-length TbBILBO1 in U-2 OS cells and probed detergent-extracted cells with the purified Nb48 (Nb48:HA:6His) and with anti-TbBILBO1 (1-110) (Fig. 8B). Nontransfected, extracted U-2 OS cells were probed with anti-TbBILBO1 (1-110) and Nb48:HA:6His and showed a negative signal (Fig. 8A). When full-length TbBILBO1 was expressed, it induced the formation of long BILBO1 polymers that were colabeled with Nb48:HA:6His and anti-TbBILBO1 (1-110) (Fig. 8B). TbBILBO1 possesses two canonical calcium-binding EF-hand domains: 1 and 2. Mutation of EF-hand domain 1 alone, or both EF-hands together, prevented the formation of linear polymers; however, mutation of EF-hand domain 2 alone (Mut2 EF-hand) resulted in the formation of helical polymers (20). We therefore used Mut2 EF-hand to test for colabeling with Nb48. Excellent colabeling was observed when the Mut2 EF-hand was expressed: the helical polymers of the mutated BILBO1 colabel with $\mathrm{Nb} 48_{\text {::HA:6His }}$ (Fig. 8C), suggesting that mutating the calcium-binding site, and preventing calcium binding, does not affect Nb48 binding. We next expressed the TbBILBO1 truncations T1, T2, T3, and T4 (refer to Fig. $2 \mathrm{G}$ ) in U-2 OS cells and used the previous anti-TbBILBO1 monoclonal IgM antibody to label T3 and T4 constructs. Those previous studies had shown that TbBILBO1 truncations T1 (aa 1 to 170) and T2 (aa 1 to 250) are soluble in U-2 OS cells and do not form polymers (20). No signal was observed when these extracted cells were probed by anti-TbBILBO1

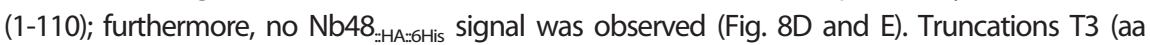
171 to 587) and T4 (aa 251 to 587), however, form long polymers when expressed in U2-OS cells, and as with FL polymers, these were positively colabeled when probed with TbBILBO1 and $\mathrm{Nb} 48_{\text {:HA:6His }}$ (Fig. 8F and G). As such, this suggests that the Nb48 epitope lies between the amino acids 251 to 587. These results illustrate that as a specific nanobody probe, Nb48:HA:6His' in a heterologous environment, performed equally to a rabbit polyclonal antibody (antiTbBILBO1 1-110) and to anti-TbBILBO1 IgM.

To determine if INb48 can bind to TbBILBO1 in the U-2 OS heterologous system and influence polymerization, we coexpressed INb48: hand, or truncated forms of TbBILBO1. Expression of INb48: 3 HA alone produced a cytoplasmic signal in whole-cell preparations (Fig. $8 \mathrm{H}$ ) that was eliminated after detergent extraction (Fig. 8I), demonstrating that INb48::3HA does not bind to any cytoskeletal structure in U-2 OS cells and does not form polymers. When INb48::3HA was coexpressed with FL-TbBILBO1 and cells were detergent extracted, a strong colocalization was observed between the TbBILBO1 signal and that of $1 \mathrm{Nb} 48_{: 3 \mathrm{HA} \text {, }}$ demonstrating that INb48::3HA was expressed and binds to TbBILBO1, in vivo, in extracted conditions (Fig. 8J and K). Importantly, however, the typical polymer structures that TbBILBO1 normally forms in U-2 OS cells (refer to Fig. 8B) were absent. Instead, dense compacted structures attached to thin minor polymers were formed. Similar dense structures were observed when Mut2-EFh was coexpressed with INb48 ${ }_{: 3 \mathrm{HA} \text {, }}$ indicating that the EF-hand domain 2 mutation (which prevents calcium binding) did not influence binding of INb48:3HA to TbBILBO1 (Fig. 8L). These data indicate that binding of INb48 ${ }_{: 3 \mathrm{HA}}$ to $\mathrm{TbBILBO} 1$ modifies and reduces linear polymerization in this environment.

When INb48 ${ }_{: 3 \mathrm{HA}}$ was coexpressed with T1 and with T2 (both have been demonstrated to be cytoplasmic in nonextracted cells) (20), a cytoplasmic signal was observed (Fig. 8M and $\mathrm{N}$ ). In contrast to the long, linear polymers observed when T3 alone was expressed (Fig. 8F), coexpression of $\mathrm{T} 3$ and INb48: $3 \mathrm{HA}$ induced dense globular structures (Fig. 8O), similar to the observations made when INb48 ${ }_{\text {:3HA }}$ was coexpressed with FL-TbBILBO1 (Fig. 8K) or Mut2EFh (Fig. 8L). Coexpression of INb48:3HA with T4 resulted in long polymers similar, but not identical, to expression of T4 alone (as observed in Fig. 8G). Instead, the INb48::3HA-bound T4 


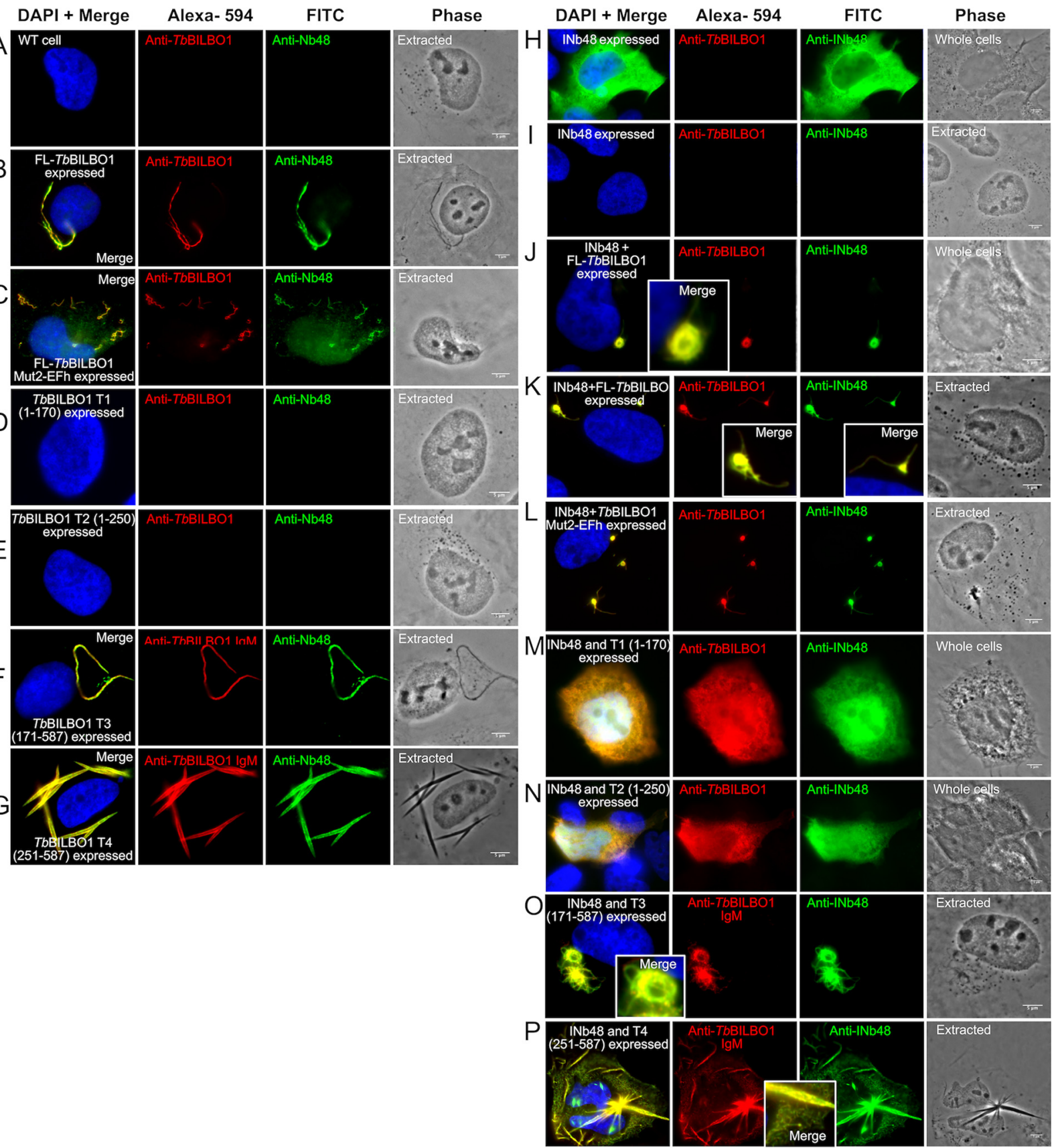

FIG $8 \mathrm{Nb} 48$ and INb48 nontransfected U-2 OS cell extracted with Triton X-100, probed with anti-TbBILBO1 (1-110 rabbit) and purified Nb48:HA::6His and revealed independently by anti-HA (mouse) and their respective secondary fluorophores: anti-rabbit, Alexa fluor 594 and anti-mouse, FITC-conjugated. No labeling is observed. (B) A U-2 OS cell that is expressing FL TbBILBO1 and probed as described above. TbBILBO1 forms polymers and Nb48: $\mathrm{HA:GHis}$ labels these polymers. (C) A U-2 OS cell expressing mutated EF-hand domain 2 (Mut2EF-TbBILBO1) and probed as described above. The typical spiral polymers of this mutant are formed and are colabeled with rabbit 1-110 and Nb48. HA:6His. (D and E) TbBILBO1 truncations T1 (aa 1 to 170) and T2 (aa 1 to 250), which are both cytoplasmic and thus eliminated during detergent extraction and thus not labeled. ( $\mathrm{F}$ and G) The typical signals observed when TbBILBO1 truncations T3 (171 to 587 aa) and T4 (251 to 587 aa) are expressed in U-2 OS; the resulting polymers formed are both positive for TbBILBO1 (in this case anti-TbBILBO1 mouse monoclonal, IgM) and Nb48:HA:: $\mathrm{HA}$ rabbit, IgG). The IgM was used here because it binds to the $C$ terminus whereas anti-TbBIL1BO (1-110) binds to the $\mathrm{N}$ terminus of TbBILBO1. (H to P) In all experiments, INb48, TbBILBO1, and TbBILBO1 mutations and truncations were expressed in U-2 OS cells for $24 \mathrm{~h}$ and then processed for IFA. IFA of nonextracted U-2 OS cell expressing INb48 ${ }_{\text {zHA }}$ only, and probed with anti-TbBILBO1 and anti-HA to detect the intrabody. This cell shows that INb48: anti-HA after detergent extraction, showing that INb48. ${ }_{\text {. }}$ has been removed after extraction. (J) Illustration of a nonextracted cell expressing $1 \mathrm{Nb} 48_{: 3 \mathrm{HA}}$ and FL-TbBILBO1 simultaneously and probed as in panels $\mathrm{H}$ and I. It illustrates that INb48 ${ }_{: 3 \mathrm{HA}}$ binds to the TbBILBO1 polymers. However, these polymers are different from WT polymers shown in panel $B$, suggesting that INb48: ${ }_{\text {:3A }}$ affects normal TbBILBO1 polymer formation. (K) A cell treated as in panel J but detergent extracted, demonstrating that INb48 ${ }_{: 3 \mathrm{HA}}$ is not removed upon detergent extraction. 
polymers were associated with many small, detergent-insoluble, network-forming polymers (Fig. 8P), which were never observed in T4-only expression as described previously (20) and suggest that binding of INb48: these data suggest a critical disturbance by INb48 in TbBILBO1 polymer organization when coexpressed in a heterologous mammalian system. IFA controls for INb48: $3 \mathrm{HA}$ and TbBILBO1 expression in U-2 OS cells alone and together can be found in Figure S4A to D, demonstrating a lack of labeling when no primary antibodies were used. Figure S5A to E shows the control intrananobody against green fluorescent protein (INbGFP) expressed in U-2 OS cells exhibiting a cytoplasmic signal which was extracted in cytoskeleton preparations and did not colocalize or bind to TbBILBO1 polymers or any other structure. Negative and no primary antibody controls are also shown.

\section{DISCUSSION}

Nanobodies are rapidly being developed, not only as revolutionary therapeutics but also as biological tools. In mammalian cells, they have been used to assess protein function intracellularly (intrabodies), to explore protein-protein interactions, and as protein inhibitors $(42,53-56)$. Nanobodies have been used in a number of experiments associated with trypanosomes, including the potential of nanobodies as diagnostic tools, in experimental therapy, and as trypanolytic agents $(42,57-59)$. However, intrabodies have not been used to target the trypanosome cytoskeleton intracellularly as a knockdown tool. This study shows that $\mathrm{Nb48}$ proved to be an excellent tool for detecting its trypanosomatid target antigen TbBILBO1 by Western blotting and by immunofluorescence (IFA) on fixed trypanosomes and immuno-electron microscopy. Further, when expressed as an intrabody, INb48 targeted specifically TbBILBO1 and functioned to disrupt the role of this essential cytoskeletal protein.

The difference in the IFA behavior between $\mathrm{Nb} 48$ and $\mathrm{Nb9}$ may be explained by their CDR3 sequences. If we consider that the two target epitopes are clearly different, then this can, in part, explain a difference in detection by IFA. This would also suggest that binding of $\mathrm{Nb} 48$ to its BILBO1 epitope is possible under the in vivo native BILBO1 expression levels within the $\mathrm{FPC}$, whereas binding of $\mathrm{Nb9}$ is impaired due to the possible presence of BILBO1 partner proteins that could partially shield the epitope or because the epitope is not fully exposed. Overexpression of BILBO1 would most probably produce and expose much more unshielded and/or accessible BILBO1 epitope allowing Nb9 to bind. This would also explain why $\mathrm{Nb9}$ was initially selected using enzyme-linked immunosorbent assay (ELISAs) since the selection protocol was based on the use of purified, denatured recombinant BILBO1.

INb48 modifies TbBILBO1 polymers in U-2 OS cells and in T. brucei. Nb48 and INb9 both bind to the T4 domain of TbBILBO1 (aa 251 to 587), demonstrating that they recognize the same epitope in vitro and in vivo. Binding of INb48 to TbBILBO1 in U-2 OS cells modifies polymer structure and induced condensed structures (Fig. $8 \mathrm{~J}$ and $\mathrm{K}$ ) compared to the long, comma-shaped, or annular-shaped polymers that are observed normally (20). INb48 binds to the domain of TbBILBO1 that includes the coiled-coil domain (CCD) and the leucine zipper (LZ) domain both involved in protein-protein interaction, and importantly,

FIG 8 Legend (Continued)

(L) The cell in this image is simultaneously expressing an EF-hand domain 2 mutated form of TbBILBO1 (Mut2-EFh) and INb48 ${ }_{: 3 \mathrm{HA}}$. The typical helical spirals formed by the Mut2-EF-hand protein are not formed when bound by INb48 affects polymer formation. TbBILBO1 proteins and INb48::3HA were expressed in U-2 OS cells for $24 \mathrm{~h}$; in this case, INb48 $: 3 \mathrm{HA}$ was coexpressed with T1 to T4 truncations. Cells were then processed for IFA using either the anti-TbBILBO1 (1-110) rabbit polyclonal, raised to and binding only the $\mathrm{N}$ terminus of TbBILBO1, for detecting T1 and T2, or an in-house-made anti-TbBILBO1 IgM mouse monoclonal, which recognizes the complement terminus of TbBILBO1; anti-HA was used to detect INb48. ${ }_{\text {. } \mathrm{HA}}$ followed by their respective secondary antibodies. (M) An IFA image of a U-2 OS cell simultaneously expressing INb48 cytoplasmic and therefore give an overlapping signal. (N) An IFA image of a U-2 OS cell simultaneously expressing INb48 ${ }_{*: 3 \mathrm{HA}}$ and TbBILBO1 T2. Similar to panel $M$, both of these proteins are cytoplasmic and give an overlapping signal. (O) This is an IFA image of a U-2 OS cell simultaneously expressing INb48 ${ }_{: 3 \mathrm{HA}}$ and TbBILBO1 T3. The typical linear filaments formed by T3 are modified when bound by INb48 ${ }_{\text {:3HA }}$ suggesting that the intrabody binding inhibits the typical polymer formation. (P) Detergent-extracted IFA images of U-2 OS cells simultaneously expressing INb48..3HA and TbBILBO1 T4. The spindle shaped polymers, as observed in Figure 8G, are observed to a lesser extent, but numerous smaller polymers are present in the cytoplasm. These are never present in cells expressing T4 alone, suggesting that the binding of INb48 to T4 modifies the polymer-forming capacity of this truncation. Scale bar is $5 \mu \mathrm{m}$. 
the CCD is required for dimerization and the $L Z$ domain is required for polymerization (20, 24). Based on the structures observed in the U-2 OS cells, both dimerization and polymerization occur, but it is difficult to speculate on how INb48 affects the polymers. However, one could expect the same effect in parasites, resulting in alteration in FPC formation. Indeed, the expression of INb48 in T. brucei led to perturbation of the structure of the FPC and the $\mathrm{HC}$, as seen by IFA and immuno-electron micrograph (iEM) labeling of TbBILBO1 and TbMORN1, respectively. Interestingly, INb48 labeled strongly the MtQ, but the typical annular FPC structure and "hook and shank" aspect of the HC were not observed in many saltextracted flagella in iEM experiments. Also, IFA experiments demonstrated (via the elongated structures labeled with an anti-BILBO1 polyclonal) that INb48 affects the biogenesis of the FPC. This suggests that TbBILBO1 and TbMORN1 traffic along the MtQ.

INb48 induces BILB01-RNAi knockdown-like phenotypes. The perturbations of TbBILBO1 assembly by INb48 led to RNAi knockdown-like phenotypes, and this included similar disruption to the endomembrane system. Although membrane trafficking was affected by INb48 expression, it does not have membrane targeting or retention signals, which are sometimes used on INbs. This suggests that the accumulation of intracellular vesicles following INb48 expression (Fig. 6) is due to disruption in endo-/exocytosis and is a downstream consequence of the disturbance of the flagellar pocket and the HC. After $24 \mathrm{~h}$ of INb48 induction, there was no INb48 or BILBO1 protein degradation and the BILBO1 RNAi-like phenotypes were apparent, but at 48-h induction there was considerable protein degradation probably due to cell lysis. INb48 binding to BILBO1 might result in BILBO1 sequestration, which could lead to intense disruption of the FP and at some point induce cell lysis. It is also possible that the intrabody binds to BILBO1 that has already been incorporated into the FPC but does not disrupt its function. If the intrabody is only able to capture free BILBO1, the difference in effect on free versus FPC-incorporated BILBO1 could explain the delay in the phenotype. In this context, it is noteworthy that we also observed dramatic changes in BILBO1 polymers when they were coexpressed with INb48 in U2-OS cells. The binding of INb48 to newly forming BILBO1 polymers could prevent correct FPC formation by inducing malformation of the growing BILBO1 polymers. Indeed, Figures 5B and D (and Fig. S3D and E) illustrate the formation of unusual, BILBO1-positive, linear polymers when INb48 was expressed in trypanosomes instead of the annular polymers normally present in a wild-type FPC. We are currently investigating the knockdown effect in more detail and also the trafficking of FPC proteins to the FPC to ascertain whether the MtQ is implicated. This may also answer, in part, the question of how the FPC, a flagellum-associated complex of proteins, is built.

In conclusion, we induced a BILBO1 RNAi phenotype not by knocking down protein expression but rather by preventing protein function. This indicates that an intrabody-induced, protein binding approach can prevent the formation of a new FPC most likely even in the presence of BILBO1 interacting proteins. This would indeed suggest that BILBO1 is the main protein performer in FPC biogenesis. Therefore, our data support the hypothesis that targeting minor, yet essential, cytoskeletal proteins is of considerable merit in the search to understand parasite biology. Intrabodies would be useful in organisms that do not have RNAi machinery and to characterize the biology of emerging pathogens, newly discovered protists, and new model organisms (48). As a consequence, the data presented here demonstrate, for the first time, the use of a functional, tractable, anti-cytoskeletal intrabody in T. brucei that is able to precisely target and bind to its target epitope and induce disruption of a cytoskeletal structure leading to rapid cell death.

\section{MATERIALS AND METHODS}

Ethics statement. The alpaca immunization was carried out by Nanobody Service Facility, VIB, Belgium, and was approved by the Ethical Committee for Animal Experiments of the Vrije Universiteit Brussel (VUB), Brussels, Belgium.

Alpaca immunization and $\mathbf{N b}$ library construction. HIS-tagged, full-length $T$. brucei brucei BILBO1 protein ( $\left.{ }_{6 \mathrm{His:}} \mathrm{TbBILBO1}\right)$ was expressed in bacteria, purified in urea on nickel nitrilotriacetic acid (Ni-NTA) resin by affinity column purification, as described in reference 25 , and sent to Nanobody Service Facility (NSF, VIB, Brussels). The nanobody library was constructed as described by NSF-VIB (Nanobody Service Facility, Belgium) and produced $2 \times 10^{8}$ independent transformants that were subjected to two rounds of panning, performed on solid-phase coated full-length TbBILBO1 antigen (10 $\mathrm{g} /$ well). Ninety-five colonies from round two were randomly selected and analyzed by ELISA for the presence of antigen-specific Nbs in their periplasmic 
extracts. Thirty-four colonies scored positive, representing seven different Nbs from three different groups based on their gene sequences: $\mathrm{Nbs}$ in each group have very similar sequence data. The $\mathrm{Nb}$ genes were cloned into a pMECS vector containing a secretion PelB leader signal sequence at the $\mathrm{N}$ terminus and a hemagglutinin $\mathrm{A}(\mathrm{HA})$ tag and a hexa-histidine (His) tag at the $C$ terminus for purification and immunodetection. These vectors were transformed into TG1 Escherichia coli and stored at $-80^{\circ} \mathrm{C}$.

Nanobody cloning and bacterial expression. Nbs were subcloned from pMECS to pHEN6c vector as follows: TG1 E. coli harboring the Nbs were grown overnight on solid agar plus ampicillin (100 $\mu \mathrm{g} / \mathrm{ml})$. The Nb genes were amplified by PCR directly on colonies using specific primers A6E (5' GAT GTG CAG CTG CAG GAG TCT GGR GGA GG 3') and PMCF (5' CTA GTG CGG CCG CTG AGG AGA CGG TGA CCT GGG T $3^{\prime}$ ). PCR fragments were digested with Pstl and Bstll and then ligated into the pHEN6c vector harbored in $\mathrm{XL} 1$ blue $E$. coli and stored at $-80^{\circ} \mathrm{C}$. For expression and purification, WK6 E. coli cells were transformed with pHEN6c harboring the Nb. PCR on transformed colonies, using M13R (5' TCA CAC AGG AAA CAG CTA TGA C $3^{\prime}$ ) and PMCF (5' CTA GTG CGG CCG CTG AGG AGA CGG TGA CCT GGG T $3^{\prime}$ ), confirmed the Nb insert. TbBILBO1, TbBILBO1 EF-hand domain mutant 2, and TbBILBO1 truncations T1, T2, T3, and T4 were expressed in the heterologous mammalian system, U2-OS, by using pCDNA3 vector as described in reference 23. $\mathrm{Nb} 48$ sequence was cloned into the pcDNA3.1 C terminus tag 3HA vector (derived from pCDNA3.1 Invitrogen) between Nhel and Xhol restriction sites and expressed transiently as an intrabody, INb48 ${ }_{: 3 \mathrm{HA}}$.

Intrananobody expression in Trypanosoma. The $\mathrm{Nb}$ inserts were digested out from pHEN6c with HindIII and Ndel and cloned into HindIII and Ndel digested pLew100-3cMyc (60). Plasmid sequences were confirmed by DNA sequencing.

Nanobody expression and purification. Freshly transformed WK6 with pHEN6c-Nb vectors was inoculated into $10 \mathrm{ml}$ LB plus ampicillin (100 $\mu \mathrm{g} / \mathrm{ml})$ plus $1 \%$ glucose and incubated overnight at $37^{\circ} \mathrm{C}$, shaking at $250 \mathrm{rpm}$. Terrific broth (TB) medium was prepared for $\mathrm{Nb}$ expression ( $2.3 \mathrm{~g} /$ liter $\mathrm{KH}_{2} \mathrm{PO}_{4}, 16.4$ $\mathrm{g} /$ liter $\mathrm{K}_{2} \mathrm{HPO}_{4} \cdot 3 \mathrm{H}_{2} \mathrm{O}, 12 \mathrm{~g} /$ /iter tryptone, $24 \mathrm{~g} /$ liter yeast extract, $4 \%$ glycerol). One milliliter of culture was added to $330 \mathrm{ml}$ TB plus $100 \mu \mathrm{g} / \mathrm{ml}$ ampicillin, $2 \mathrm{mM} \mathrm{MgCl}_{2}$, and $0.1 \%$ glucose, incubated at $37^{\circ} \mathrm{C}$, shaking at $250 \mathrm{rpm}$, until a mid-log phase of growth optical density at $600 \mathrm{~nm}\left(\mathrm{OD}_{600}\right)$ of 0.6 to 0.9 was reached. $\mathrm{Nb}$ expression was induced with $1 \mathrm{mM}$ isopropyl $\beta$-D-1-thiogalacto-pyranoside (IPTG). The culture was incubated for $17 \mathrm{~h}$ at $28^{\circ} \mathrm{C}$ with shaking. $\mathrm{Nb}$ was extracted from the periplasm as follows. Cultures were centrifuged for $16 \mathrm{~min}$ at $4,500 \times g$. The pellet was resuspended in $4 \mathrm{ml}$ of TES $(0.2 \mathrm{M}$ Tris [pH 8.0], $0.5 \mathrm{mM}$ EDTA, $0.5 \mathrm{M}$ sucrose) and then shaken for $1 \mathrm{~h}$ on ice at $110 \mathrm{rpm}$. Four milliliters of TES/4 (TES diluted 1:4 in water) was added and further incubated on ice for $1 \mathrm{~h}$ with shaking and then centrifuged for $60 \mathrm{~min}$ at $4,500 \times g$ at $4^{\circ} \mathrm{C}$ to release the contents of the periplasmic space (61). The supernatant contains the periplasmic extract with $\mathrm{Nb}$. A control periplasmic extraction was performed using the same procedure as above, without the $\mathrm{Nb}$ insert in the vector.

The periplasmic extract was filtered on $0.45 \mu \mathrm{m}$ and loaded onto a HisTrap FF column (GE Healthcare) preequilibrated with running buffer (phosphate-buffered saline [PBS], $20 \mathrm{mM}$ imidazole, 10\% glycerol, protease inhibitor cocktail set III, Calbiochem, 539134-1 at 1:10,000 dilution). The column was washed with five column volumes (CV) of lysis running buffer, and bound protein was eluted by $500 \mathrm{mM}$ imidazole/running buffer with $10 \mathrm{CV}$. Elutions containing the highest concentration of purified nanobody were pooled and dialyzed against $1 \times$ PBS plus 20\% glycerol using Slide-A-Lyzer (Thermo Scientific B2162132) and stored in $1 \times$ PBS plus $20 \%$ glycerol. To verify the presence of $\mathrm{Nb}$ in purified elutions, $10 \mu \mathrm{l}$ from each elution was run on 15\% SDS-PAGE gel stained with Coomassie brilliant blue (Instant Blue Expedeon Ltd.). Proteins from a second identically loaded gel were transferred to polyvinylidene difluoride (PVDF) membrane using a semidry transfer method (BIORAD), and Western blotting was performed using anti-His (Sigma $\mathrm{H}-1029$, mouse, 1:3,000) and anti-HA (Biolegend MMS-101R or Santa-Cruz 7392, 1:1,000) antibodies to detect the Nb, followed by anti-mouse horseradish peroxidase (HRP) conjugated (Jackson, 115-035-044, 1:10,000 or Jackson, 515-035-062, 1:1,000).

Surface plasmon resonance assays. Surface plasmon resonance (SPR) was used to measure the binding affinity of the nanobodies to TbBILBO1 purified protein, the benefits being that it is measured in real-time and is label free. All solutions and buffers used were filtered and degassed. The experiments were performed at $25^{\circ} \mathrm{C}$ using a Biacore T200 instrument (GE Healthcare Life Sciences, Uppsala, Sweden) flowed with EP+ buffer (10 mM HEPES [pH 7.4], $150 \mathrm{mM} \mathrm{NaCl}, 3 \mathrm{mM}$ EDTA, 0.05\% Tween 20) from the manufacturer as running buffer. For TbBILBO1 expression, 6His:: TbBILBO1 protein was overexpressed in bacteria and soluble supernatant was purified on Ni-NTA resin as described in reference 13. Briefly, the cells were grown at $37^{\circ} \mathrm{C}$ in Luria-Bertani (LB) medium containing $50 \mu \mathrm{g} / \mathrm{ml}$ kanamycin to an $\mathrm{OD}_{600}$ of 0.6. The cells were cold-shocked on ice for $30 \mathrm{~min}$ and induced with $1 \mathrm{mM}$ isopropyl- $\beta$-D-thiogalactopyranosid (IPTG) for $2 \mathrm{~h}$ at $16^{\circ} \mathrm{C}$. After centrifugation, the cell pellet was lysed by sonication in lysis buffer (2 mM Tris- $\mathrm{HCl}$ [pH 7.4], $0.5 \mathrm{M} \mathrm{NaCl}, 20 \mathrm{mM}$ imidazole, $10 \%$ glycerol, protease inhibitor cocktail set III, Calbiochem, 1:10,000) and centrifuged $\left(10,000 \times g, 30 \mathrm{~min}, 4^{\circ} \mathrm{C}\right)$ to remove cell debris. The supernatant was filtered at $0.45 \mu \mathrm{m}$ and loaded onto a HisTrapTMFF column (GE Healthcare) preequilibrated with the same lysis buffer. The column was washed with 5 column volumes (CV) of lysis buffer, and bound protein was eluted by $500 \mathrm{mM}$ imidazole with $10 \mathrm{CV}$ in lysis buffer. TbBILBO1 fractions were pooled and dialyzed against PBS and $10 \%$ glycerol, and concentration was measured using Thermo Scientific NanoDrop 2000. TbBILBO1, prepared at $50 \mu \mathrm{g} / \mathrm{ml}$ in $10 \mathrm{mM}$ sodium acetate buffer (pH 7) and was immobilized on one flow cell of a CM5 sensor chip (GE Healthcare, lot no. 10266084) by amine coupling as indicated by the manufacturer, and the protein solution was injected for $11 \mathrm{~min} ; 2,973$ resonance units $(\mathrm{RU})$ of protein were immobilized. One flow cell was left blank for double-referencing of the sensorgrams. The nanobodies were dialyzed against the EP+ running buffer and were injected over the target by single-cycle kinetics (SCK). This method consists of injecting the partner sequentially at increasing concentrations without a regeneration step between each concentration injected. Protein concentrations were determined using a Thermofisher Nanodrop One spectrophotometer (Ozyme) and were injected over the target by single-cycle 
kinetics (SCK) $(62,63)$. The surface was regenerated after each SCK cycle with two 1-min pulses of $10 \mathrm{mM}$ glycine- $\mathrm{HCl}(\mathrm{pH}$ 2.1). The sensorgrams were analyzed with Biacore T200-v2.0 evaluation software using a Langmuir 1:1 model of interaction to determine the association $\left(k_{a}\right)$ and dissociation $\left(k_{b}\right)$ rate constants. A regeneration test was performed using $10 \mathrm{mM}$ glycine- $\mathrm{HCl}(\mathrm{pH} 2.1$; $\mathrm{GE}$ health care), $10 \mathrm{mM}$ glycine- $\mathrm{HCl}(\mathrm{pH}$ 2.1), at $30 \mu \mathrm{g} / \mathrm{min}$ for $30 \mathrm{~s}$ with TbBILBO1 alone to ensure no loss of signal. The dissociation equilibrium constant, $K_{D}$, was calculated as $k_{b} / k_{a}$. The values shown are the average and standard deviation of three independent experiments with samples injected in duplicate.

Trypanosome cell lines, culture, and transfection. Procyclic (PCF) cell line Tb427 29.13 Trypanosoma brucei brucei was grown in SDM-79 medium (pH 7.4; GE Healthcare, G3344-3005) supplemented with $2 \mathrm{mg} / \mathrm{ml}$ hemin (Sigma-Aldrich, H-5533), 10\% fetal bovine serum (FBS) complement deactivated at $56^{\circ} \mathrm{C}$ for $30 \mathrm{~min}$ (Gibco, 11573397), and incubated at $27^{\circ} \mathrm{C}$. Selection antibiotics hygromycin $(25 \mu \mathrm{g} / \mathrm{ml})$ and neomycin $(10 \mu \mathrm{g} / \mathrm{ml})$ were added to the media to maintain the plasmid vectors pLew29 and pLew13 (60). For transfection, the pLew100_Nb_3cMyc plasmids were linearized with Notl and then concentrated and purified by ethanol precipitation. Laboratory cell lines PCF Tb427 29.13 were grown to $5 \times 10^{6}$ to $10 \times 10^{6} \mathrm{cells} / \mathrm{ml}$, and then $3 \times 10^{6}$ cells were electro-transfected with $10 \mu \mathrm{g}$ of plasmid using the program X-001 of the Nucleofectorll, AMAXA apparatus (Biosystems) as described in reference 60 with the transfection buffer described therein. After transfection, clones were obtained by serial dilution and maintained in logarithmic phase growth at $2 \times 10^{6}$ cells $/ \mathrm{ml}$. Selection antibiotics hygromycin $(25 \mu \mathrm{g} / \mathrm{ml})$ and neomycin $(10 \mu \mathrm{g} / \mathrm{ml})$ were added to the media to maintain the laboratory cell lines Tb427 29.13 (procyclic forms [PCF]). Phleomycin (5 $\mu \mathrm{g} / \mathrm{ml}$ ) was added to the media of transfected cells to select for those harboring the pLew100-3cMyc vector and the $\mathrm{Nb}$ gene. Expression of $\mathrm{Nb}$ in the parasites (intrabody) was induced with tetracycline at $0.1 \mathrm{ng} / \mathrm{ml}$ to $1 \mu \mathrm{g} / \mathrm{ml}$ tetracycline. Growth curves representing logarithmic number of cells were calculated by counting the number of cells every $24 \mathrm{~h}$ using Malassez counting chamber slides. Error bars represent standard error of the mean (SEM).

Mammalian cell line culture and transfection. U-2 OS cells (human bone osteosarcoma epithelial cells, ATCC no. HTB-96) (64) were grown in Dulbecco's modified Eagle medium (DMEM) Glutamax (Gibco) supplemented with final concentrations of 10\% fetal calf serum (Invitrogen), 100 units/ml of penicillin (Invitrogen), and $0.100 \mathrm{mg} /$ liter of streptomycin (Invitrogen) at $37^{\circ} \mathrm{C}$ plus $5 \% \mathrm{CO}_{2}$. Exponentially growing U-2 OS cells in 24-well plate with glass coverslips were lipotransfected as described in reference 20 with $0.5 \mu \mathrm{g}$ of DNA using lipofectamine 2000 in OPTIMEM (Invitrogen) according to the manufacturer's instructions and processed for immunofluorescence $24 \mathrm{~h}$ posttransfection.

Western blotting. Trypanosomes were collected, centrifuged at $800 \times g$ for $10 \mathrm{~min}$, and washed in $1 \times$ PBS. For whole-cell samples, the number of trypanosomes to be loaded per well was calculated and then resuspended in protein sample buffer $2 \times$ plus nuclease $250 \mathrm{IU} / \mathrm{ml}$ (Sigma-Aldrich, E1014). The samples were boiled at $100^{\circ} \mathrm{C}$ for $5 \mathrm{~min}$ and then stored at $-20^{\circ} \mathrm{C}$ until required. We prepared $15 \%$ SDS-PAGE gels and loaded samples with $5 \times 10^{6}$ WT PCF trypanosome cells or PCF expressing pLew100-Nb-3cMyc (induced with tetracycline $1 \mu \mathrm{g} / \mathrm{ml}, 24$ to $48 \mathrm{~h}$ ). For detection of $\mathrm{Nb}$ in bacterial periplasmic extract, $2 \times 10^{8}$ bacterial cells or equivalent volume of periplasmic extract, flowthrough, or elution were loaded. Samples were separated according to the manufacturer's recommendations and then transferred in a semidry system (BIORAD trans-blot semidry transfer cell 221BR54560) onto PVDF membrane and incubated with blocking solution (BS; 5\% skimmed milk powder, $0.2 \%$ Tween 20 in $1 \times$ Tris-buffered saline [TBS]) for 60 min. For detection of intrabody, INb:: ${ }_{3 \mathrm{cmyc}}$ expression in trypanosomes, an anti-cMyc antibody (Sigma, rabbit, C-3956) was diluted in blocking solution 1:1,000 and incubated with the membranes overnight at $4^{\circ} \mathrm{C}$. Membranes were washed in $1 \times$ TBS and then in BS before probing with anti-rabbit antibody conjugated to horseradish peroxidase (HRP) (1:10,000 in BS, Sigma, goat, A9169) for $60 \mathrm{~min}$ at room temperature (RT). The following antibodies were also used as primaries: anti-TbBILBO1 aa 1-110 (rabbit polyclonal, 1:1,000) (23). Anti-TbEnolase (rabbit polyclonal, 1:25,000) (65) and anti-TAT1 (anti-tubulin mouse monoclonal, 1:1,000) (66) were used as loading controls. A secondary anti-mouse HRP antibody was also used, diluted 1:10,000 (Jackson, sheep, 515-035-062). Revelation was carried out using Image Quant LAS 4000 (GE) or Chemidoc (BioRad). Purified Nbs were used as primary antibodies in Western blotting to detect the presence of TbBILBO1 in whole trypanosome cells. Whole-cell extract of $5 \times 10^{6} \mathrm{WT}$ PCF trypanosome cells, or PCF expressing TbBILBO1-3cMyc, full-length or truncations of TbBILBO1 (T1, T2, T3, and T4, induced with tetracycline $1 \mu \mathrm{g} / \mathrm{ml}, 24 \mathrm{~h}$ ), were loaded on 12 or 15\% SDS-PAGE. Western blotting was carried out as described above, and the purified $\mathrm{Nb}_{-3 \mathrm{HA}: 6 \mathrm{His}}$ was used at a concentration of $10 \mu \mathrm{g} / \mathrm{ml}$ to probe the membranes. To detect purified nanobody, an anti-HA (1:1,000, Biolegend, 901513) or anti-His (1:10,000, Sigma H1029) was used, followed by an anti-mouse HRP conjugated secondary (see above).

Immunofluorescence assay. One milliliter of log-phase trypanosomes was collected, centrifuged for $5 \mathrm{~min}$ at 1,000 $\times \mathrm{g}$, and washed in $1 \times$ PBS, and then $20 \mu \mathrm{l}$ was loaded onto poly-L-lysine $0.1 \%$ solution (P8920 Sigma-Aldrich) coated slides for $4 \mathrm{~min}$ to adhere. Whole cells were fixed in methanol at $-20^{\circ} \mathrm{C}$ for at least $60 \mathrm{~min}$. Cytoskeleton extraction was with $0.25 \% \mathrm{NP}-40$ in PIPES [piperazine-N,N'-bis 2ethanesulfonic acid] buffer (100 mM PIPES [pH 6.8], $1 \mathrm{mM} \mathrm{MgCl}_{2}$ ) for $5 \mathrm{~min}$ and washed twice in PIPES buffer, and cytoskeletons were fixed with 1\% paraformaldehyde (PFA) or methanol. Fixed trypanosomes were incubated with single or combination antibodies, anti-cMyc (monoclonal antibody lgG1 9E10, 1:20) to detect expressed INb, anti-TbBILBO1 (amino acids 1-110, rabbit, 1517028 bleed 1, 1:4,000), antiTbMORN1 (rabbit 1:5,000), anti-TbPFR2 (rabbit 1:200), and anti-TbBLD10 (rabbit 1:2,000) for $60 \mathrm{~min}$, washed twice in $1 \times$ PBS, and then incubated with secondary antibodies: anti-mouse IgG fluorescein isothiocyanate (FITC)-conjugated (Jackson, 115-095-164) and anti-rabbit Alexa fluor 594-conjugated (Invitrogen, A-11012). Kinetoplasts and nuclei were stained with $10 \mu \mathrm{l}$ DAPI (4',6-diamidino-2-phenylindole; $10 \mu \mathrm{g} / \mathrm{ml}$ ) in $1 \times$ PBS for $2 \mathrm{~min}$, and slides were mounted with Slowfade Gold antifade reagent (Invitrogen, Molecular Probes). Images were acquired on a Zeiss Axioimager Z1 using MetaMorph software and a Roper CoolSNAP HQ2 camera. For Nbs used as probes in IFA of fixed trypanosomes, PCF Tb427 29.13 wild type (WT) and Tb427 29.13 pLew100-TbBILBO1-3cMyc were fixed (as described above) 
and incubated with $\mathrm{Nb}(0.25 \mathrm{mg} / \mathrm{ml})$ for $1 \mathrm{~h}$, followed by anti-HA (Biolegend, 901513, mouse) diluted 1:200. For colabeling with TbBILBO1, anti-TbBILBO1 (1-110) was used diluted 1:4,000 in $1 \times$ PBS. Secondary antibodies were used with 1:100 dilution: anti-mouse FITC-conjugated (Sigma, F2012), antirabbit Alexa fluor 594-conjugated (Invitrogen, A11012). For IFA on U-2 OS cells, cells were fixed and processed as described in references 23 and 25. The primary antibodies anti-TbBILBO1 (1-110), 1:4,000 dilution, anti-TbBILBO1 (IgM) mouse monoclonal, 5F2B3 (13), 1:2 dilution, anti-HA epitope tag (mouse monoclonal, Biolegend, 1:1,000), and anti-HA (rabbit, GeneTex GTX115044, 1:1,000) were incubated for 1 $\mathrm{h}$ in a dark, moist chamber. After two $1 \times$ PBS washes, cells were incubated for $1 \mathrm{~h}$, respectively, with the secondary antibodies goat anti-rabbit lgG $(\mathrm{H}+\mathrm{L})$ conjugated to Alexa fluor 594 (Molecular Probes A11012, 1:400), goat anti-mouse IgM conjugated to Alexa fluor 594 (Invitrogen, 1:400), goat anti-mouse whole molecule conjugated to FITC (Sigma F-2012, 1:400), and goat anti-rabbit IgG $(\mathrm{H}+\mathrm{L})$ conjugated to Alexa fluor 594 (Molecular Probes A-11012, 1:400). Purified Nb48::HA:6His raised against TbBILBO1 was used as a probe $(10 \mu \mathrm{g} / \mathrm{ml})$ and incubated for $1 \mathrm{~h}$ in a dark, moist chamber. After two PBS washes, cells were incubated for $1 \mathrm{~h}$ with the secondary antibody directed against the HA tag, anti-HA epitope tag (Biolegend, 1:1,000), and then washed twice in PBS and incubated $1 \mathrm{~h}$ with the tertiary antibody goat antimouse whole molecule conjugated to FITC (Sigma, 1:400). The intrabody anti-green fluorescent protein (GFP) nanobody $3 \times \mathrm{HA}_{\text {-tagged (INbGFP }: 3 \mathrm{HA}}$ ) was probed with anti-HA mouse lgG Biolegend, diluted 1:1,000 in PBS fetal calf serum (FCS) $10 \%$, saponin $0.1 \%$, and probed with anti-mouse goat whole molecule FITC, Sigma, diluted 1:100 in PBS FCS $10 \%$, saponin $0.1 \%$. The nuclei were stained with DAPI $(0.25 \mu \mathrm{g} / \mathrm{ml}$ in PBS for $5 \mathrm{~min})$. Slides were washed and mounted with Prolong (Molecular Probes S-36930). Images were acquired on a Zeiss Imager Z1 microscope with Zeiss $63 \times$ objective (NA 1.4) using a Photometrics Coolsnap HQ2 camera and Metamorph software (Molecular Devices) and processed with ImageJ (NIH).

Flagella preparation for IFA. Ten milliliters of mid-log PCF cells were collected and washed once in $1 \times$ PBS for $5 \mathrm{~min}$ at $1,000 \times g$ at RT. Cells were extracted with $1 \mathrm{ml} \mathrm{1 \%} \mathrm{NP-40} \mathrm{in} 100 \mathrm{mM}$ PIPES (pH 6.9), $1 \mathrm{mM} \mathrm{MgCl}_{2}$ for $7 \mathrm{~min}$ at RT to make cytoskeletons. Cytoskeletons were centrifuged for $10 \mathrm{~min}$ at 5,000 $\times g$ at $4^{\circ} \mathrm{C}$ and further extracted for 15 to $30 \mathrm{~min}$ on ice with $1 \% \mathrm{NP}-40$ in $100 \mathrm{mM} \mathrm{PIPES}$ buffer plus $2 \mathrm{mM} \mathrm{MgCl}_{2}$, containing $1 \mathrm{M} \mathrm{KCl}$. Flagella were centrifuged for $5 \mathrm{~min}$ at $5,000 \times g$ at $4^{\circ} \mathrm{C}$, the supernatant was removed, and flagella were resuspended in $500 \mu \mathrm{l}$ PIPES buffer. Flagella were washed twice in $100 \mathrm{mM}$ PIPES buffer plus $1 \mathrm{mM} \mathrm{MgCl} 2$ for $5 \mathrm{~min}$ at 5,000 $\times g$ at $4^{\circ} \mathrm{C}$ before being deposed on poly-lysine coated slides and left to adhere for 5 to $10 \mathrm{~min}$. The flagella were fixed at $-20^{\circ} \mathrm{C}$ in $\mathrm{MeOH}$ or in $3 \%$ PFA as described above.

Transmission electron microscopy. Ten milliliters of mid-log-phase procyclic, Tb427 29.13 wildtype (WT) cells, pLew100-Nb48-3cMyc (induced for 24 h) was fixed, dehydrated, and embedded, cut, and stained as in reference 12 , with the exception that tannic acid was omitted and cells were initially fixed in their culture medium by the addition of glutaraldehyde to a final concentration of $2.5 \%$ and paraformaldehyde at $3.7 \%$ for $2 \mathrm{~h}$ and then pelleted at $1,000 \times g$ and fixed for $2 \mathrm{~h}$ in $2.5 \%$ glutaraldehyde in phosphate buffer (pH 7.4).

Immuno-electron microscopy. Ten milliliters of mid-log-phase $\sim 5 \times 10^{6} / \mathrm{ml} \mathrm{pLew100-Nb48-3cMyc}$ cells was induced with $1 \mu \mathrm{g} / \mathrm{ml}$ tetracycline for $24 \mathrm{~h}$. Five milliliters of induced cells was harvested at $1,000 \times g$ for $5 \mathrm{~min}$, washed twice with PBS by centrifugation $(1,000 \times g)$, and resuspended in $500 \mu l$ of PBS. Freshly glow-discharged, Formvar and carbon-coated G200-Ni grids (EMS) were floated on the droplets, and the cells were allowed to adhere for 15 min. Grids were then moved onto a drop of $1 \%$ NP-40 in PEME buffer (100 mM PIPES [pH 6.8], 1 mM MgCl $2,0.1 \mathrm{mM} \mathrm{EGTA)} \mathrm{(5} \mathrm{min,} \mathrm{RT),} \mathrm{followed} \mathrm{by} \mathrm{incu-}$ bation on a $500 \mu \mathrm{l}$ drop of $1 \% \mathrm{NP}-40,1 \mathrm{M} \mathrm{KCl}$ in PEME buffer for $15 \mathrm{~min}\left(3 \times 5 \mathrm{~min}, 4^{\circ} \mathrm{C}\right)$. Flagella were washed twice $(2 \times 5 \mathrm{~min})$ in PEME buffer at RT, equilibrated, and blocked on $50 \mu \mathrm{l}$ drops of $2 \%$ fish skin gelatin (Sigma-Aldrich G7041) or 0.5\% BSA, 0.1\% Tween 20 in PBS, incubated in $25 \mu$ l of primary antibody diluted in blocking buffer (each primary antibody was used either alone or mixed with a second primary antibody), $1 \mathrm{~h}$ at room temperature (RT). Anti-cMyc lgG mouse monoclonal antibody (9E10, purified, a kind gift from Klaus Ersfeld) was diluted 1:10 or protein G purified/concentrated supernatant (diluted 1:50). Anti-TbBILBO1 (1-110) was diluted 1:200 or 1:400. Grids were blocked and incubated in secondary antibody for $1 \mathrm{~h}$ at RT (anti-mouse goat GMTA $5 \mathrm{~nm}$ gold, and/or anti-rabbit goat $15 \mathrm{~nm}$ gold GAR15, BBInternational) 1:10 in $0.2 \%$ fish skin gelatin in PBS. Grids were blocked and fixed in $2.5 \%$ glutaraldehyde in $0.2 \%$ fish skin gelatin in PBS. Samples were negatively stained with $1 \%$ aurothioglucose (Sigma-Aldrich) (10 $\mu \mathrm{l}$ for $30 \mathrm{~s}$ ). Micrographs were taken on a Phillips Technai 12 transmission electron microscope at $100 \mathrm{kV}$.

\section{SUPPLEMENTAL MATERIAL}

Supplemental material is available online only.

SUPPLEMENTAL FILE 1, PDF file, 7.3 MB.

\section{ACKNOWLEDGMENTS}

We thank our lab members for insightful comments on the manuscript, Brooke Morriswood (University of Wurzburg, Germany) for the anti-TbMORN1 antibody, Keith Gull (University of Oxford, England) for the anti-TAT1 (anti-tubulin) antibody, Zyin Li (University of Texas, USA) for the anti-TbBLD10 antibody, Klaus Ersfeld (University of Bayreuth, Germany) for the anti-cMyc (9E10) antibody, Frédéric Bringaud (University of Bordeaux, Bordeaux, France) for the antienolase antibody, and Nicolas Biteau (University of Bordeaux, Bordeaux, France) for the antiTbPFR2 rabbit polyclonal antibody. The Robinson lab acknowledges support by the Centre 
National de la Recherche Scientifique (CNRS; https://www.cnrs.fr/) and the Université de Bordeaux (https://www.u-bordeaux.fr/). C.B.R. and M.R.R. were supported by fellowships from the French Ministry of Higher Education, Research and Innovation (https://www .enseignementsup-recherche.gouv.fr/). We acknowledge funding from the Fondation pour le Recherche Médicale (FRM, https://www.frm.org/) (FRM) grant number FDT202001010783 awarded to M.R.R. This work was also supported by Laboratoire d'Excellence (https://www .enseignementsup-recherche.gouv.fr/cid51355/laboratoires-d-excellence.html) through the LabEx ParaFrap (grant number ANR-11-LABX-0024) awarded to D.R.R. and ANR-FWF PRCI [ANR-20-CE91-0003] to M.B.

\section{REFERENCES}

1. Diall O, Cecchi G, Wanda G, Argilés-Herrero R, Vreysen MJB, Cattoli G, Viljoen GJ, Mattioli R, Bouyer J. 2017. Developing a progressive control pathway for African animal trypanosomosis. Trends Parasitol 33:499-509. https://doi.org/10.1016/j.pt.2017.02.005.

2. Simarro PP, Cecchi G, Franco JR, Paone M, Diarra A, Priotto G, Mattioli RC, Jannin JG. 2015. Monitoring the progress towards the elimination of gambiense human African trypanosomiasis. PLoS Negl Trop Dis 9:e0003785. https://doi.org/10.1371/journal.pntd.0003785.

3. Mesu VKBK, Kalonji WM, Bardonneau C, Mordt OV, Blesson S, Simon F, Delhomme S, Bernhard S, Kuziena W, Lubaki J-PF, Vuvu SL, Ngima PN, Mbembo HM, llunga M, Bonama AK, Heradi JA, Solomo JLL, Mandula G, Badibabi LK, Dama FR, Lukula PK, Tete DN, Lumbala C, Scherrer B, StrubWourgaft N, Tarral A. 2018. Oral fexinidazole for late-stage African Trypanosoma brucei gambiense trypanosomiasis: a pivotal multicentre, randomised, non-inferiority trial. Lancet 391:144-154. https://doi.org/10.1016/ S0140-6736(17)32758-7.

4. Deeks ED. 2019. Fexinidazole: first global approval. Drugs 79:215-220. https://doi.org/10.1007/s40265-019-1051-6.

5. Keating J, Yukich JO, Sutherland CS, Woods G, Tediosi F. 2015. Human African trypanosomiasis prevention, treatment and control costs: a systematic review. Acta Trop 150:4-13. https://doi.org/10.1016/j.actatropica.2015.06.003.

6. Scott V, Sherwin T, Gull K. 1997. Gamma-tubulin in trypanosomes: molecular characterisation and localisation to multiple and diverse microtubule organising centres. J Cell Science 110:157-168. https://doi.org/10.1242/ jcs.110.2.157.

7. Bastin P, Sherwin T, Gull K. 1998. Paraflagellar rod is vital for trypanosome motility. Nature 391:548. https://doi.org/10.1038/35300.

8. Bastin P, Pullen TJ, Sherwin T, Gull K. 1999. Protein transport and flagellum assembly dynamics revealed by analysis of the paralysed trypanosome mutant snl-1. J Cell Science 112:3769-3777. https://doi.org/10 .1242/jcs.112.21.3769.

9. Kohl L, Sherwin T, Gull K. 1999. Assembly of the paraflagellar rod and the flagellum attachment zone complex during the Trypanosoma brucei cell cycle. J Eukaryot Microbiol 46:105-109. https://doi.org/10.1111/j.1550 -7408.1999.tb04592.x.

10. Moreira-Leite FF, Sherwin T, Kohl L, Gull K. 2001. A trypanosome structure involved in transmitting cytoplasmic information during cell division. Science 294:610-612. https://doi.org/10.1126/science.1063775.

11. Robinson DR, Gull K. 1991. Basal body movements as a mechanism for mitochondrial genome segregation in the trypanosome cell cycle. Nature 352:731-733. https://doi.org/10.1038/352731a0.

12. Pradel LC, Bonhivers M, Landrein N, Robinson DR. 2006. NIMA-related kinase TbNRKC is involved in basal body separation in Trypanosoma brucei. J Cell Sci 119:1852-1863. https://doi.org/10.1242/jcs.02900.

13. Bonhivers M, Nowacki S, Landrein N, Robinson DR. 2008. Biogenesis of the trypanosome endo-exocytotic organelle is cytoskeleton mediated. PLoS Biol 6:e105. https://doi.org/10.1371/journal.pbio.0060105.

14. Henley GL, Lee CM, Takeuchi A. 1978. Electron microscopy observations on Trypanosoma brucei: freeze-cleaving and thin-sectioning study of the apical part of the flagellar pocket. Z Parasitenkd 55:181-187. https://doi .org/10.1007/BF00390368.

15. Webster P, Russell DG. 1993. The flagellar pocket of trypanosomatids. Parasitol Today 9:201-206. https://doi.org/10.1016/0169-4758(93)90008-4.

16. Morgan GW, Hall BS, Denny PW, Carrington M, Field MC. 2002. The kinetoplastida endocytic apparatus. Part I: a dynamic system for nutrition and evasion of host defences. Trends Parasitol 18:491-496. https://doi.org/10 .1016/s1471-4922(02)02391-7.
17. Field MC, Carrington M. 2009. The trypanosome flagellar pocket. Nat Rev Microbiol 7:775-786. https://doi.org/10.1038/nrmicro2221.

18. Webster P. 1989. Endocytosis by African trypanosomes. I. Three-dimensional structure of the endocytic organelles in Trypanosoma brucei and T. congolense. Eur J Cell Biol 49:295-302.

19. Lacomble S, Vaughan S, Gadelha C, Morphew MK, Shaw MK, McIntosh JR, Gull K. 2009. Three-dimensional cellular architecture of the flagellar pocket and associated cytoskeleton in trypanosomes revealed by electron microscope tomography. J Cell Sci 122:1081-1090. https://doi.org/10.1242/jcs.045740.

20. Florimond C, Sahin A, Vidilaseris K, Dong G, Landrein N, Dacheux D, Albisetti A, Byard EH, Bonhivers M, Robinson DR. 2015. BILBO1 is a scaffold protein of the flagellar pocket collar in the pathogen Trypanosoma brucei. PLoS Pathog 11:e1004654. https://doi.org/10.1371/journal.ppat.1004654.

21. Isch C, Majneri P, Landrein N, Pivovarova Y, Lesigang J, Lauruol F, Robinson DR, Dong G, Bonhivers M. 2021. Structural and functional studies of the first tripartite protein complex at the Trypanosoma brucei flagellar pocket collar. PLoS Pathog 17:e1009329. https://doi.org/10.1371/journal.ppat.1009329.

22. Albisetti A, Florimond C, Landrein N, Vidilaseris K, Eggenspieler M, Lesigang J, Dong G, Robinson DR, Bonhivers M. 2017. Interaction between the flagellar pocket collar and the hook complex via a novel microtubule-binding protein in Trypanosoma brucei. PLoS Pathog 13:e1006710. https://doi.org/10.1371/ journal.ppat.1006710.

23. Vidilaseris K, Morriswood B, Kontaxis G, Dong G. 2014. Structure of the TbBILBO1 protein N-terminal domain from Trypanosoma brucei reveals an essential requirement for a conserved surface patch. J Biol Chem 289: 3724-3735. https://doi.org/10.1074/jbc.M113.529032.

24. Vidilaseris K, Shimanovskaya E, Esson HJ, Morriswood B, Dong G. 2014. Assembly mechanism of Trypanosoma brucei BILBO1, a multidomain cytoskeletal protein. J Biol Chem 289:23870-23881. https://doi.org/10.1074/jbc .M114.554659.

25. Vidilaseris K, Landrein N, Pivovarova Y, Lesigang J, Aeksiri N, Robinson DR, Bonhivers M, Dong G. 2020. Crystal structure of the N-terminal domain of the trypanosome flagellar protein BILBO1 reveals a ubiquitin fold with a long structured loop for protein binding. J Biol Chem 295:1489-1499. https://doi.org/10.1074/jbc.RA119.010768.

26. Sherwin T, Gull K. 1989. The cell division cycle of Trypanosoma brucei brucei: timing of event markers and cytoskeletal modulations. Philos Trans $\mathrm{R}$ Soc Lond B Biol Sci 323:573-588. https://doi.org/10.1098/rstb.1989.0037.

27. Dong X, Lim TK, Lin Q, He CY. 2020. Basal body protein TbSAF1 is required for microtubule quartet anchorage to the basal bodies in Trypanosoma brucei. mBio 11:e00668-20. https://doi.org/10.1128/mBio.00668-20.

28. Esson HJ, Morriswood B, Yavuz S, Vidilaseris K, Dong G, Warren G. 2012. Morphology of the trypanosome bilobe, a novel cytoskeletal structure. Eukaryot Cell 11:761-772. https://doi.org/10.1128/EC.05287-11.

29. Hamers-Casterman C, Atarhouch T, Muyldermans S, Robinson G, Hamers C, Songa EB, Bendahman N, Hamers R. 1993. Naturally occurring antibodies devoid of light chains. Nature 363:446-448. https://doi.org/10.1038/363446a0.

30. Greenberg AS, Avila D, Hughes M, Hughes A, McKinney EC, Flajnik MF. 1995. A new antigen receptor gene family that undergoes rearrangement and extensive somatic diversification in sharks. Nature 374:168-173. https://doi.org/10 $.1038 / 374168 \mathrm{a} 0$.

31. Holliger P, Hudson PJ. 2005. Engineered antibody fragments and the rise of single domains. Nat Biotechnol 23:1126-1136. https://doi.org/10.1038/nbt1142.

32. Ward ES, Güssow D, Griffiths AD, Jones PT, Winter G. 1989. Binding activities of a repertoire of single immunoglobulin variable domains secreted from Escherichia coli. Nature 341:544-546. https://doi.org/10.1038/341544a0. 
33. Muyldermans S. 2013. Nanobodies: natural single-domain antibodies. Annu Rev Biochem 82:775-797. https://doi.org/10.1146/annurev-biochem -063011-092449.

34. Kaplon H, Reichert JM. 2019. Antibodies to watch in 2019. MAbs 11:219-238. https://doi.org/10.1080/19420862.2018.1556465.

35. Southwell AL, Ko J, Patterson PH. 2009. Intrabody gene therapy ameliorates motor, cognitive, and neuropathological symptoms in multiple mouse models of Huntington's disease. J Neurosci 29:13589-13602. https://doi.org/10.1523/JNEUROSCI.4286-09.2009.

36. Zhou C, Przedborski S. 2009. Intrabody and Parkinson's disease. Biochim Biophys Acta 1792:634-642. https://doi.org/10.1016/j.bbadis.2008.09.001.

37. Van Impe K, Bethuyne J, Cool S, Impens F, Ruano-Gallego D, De Wever O, Vanloo B, Van Troys M, Lambein K, Boucherie C, Martens E, Zwaenepoel O, Hassanzadeh-Ghassabeh G, Vandekerckhove J, Gevaert K, Fernández LÁ, Sanders NN, Gettemans J. 2013. A nanobody targeting the F-actin capping protein CapG restrains breast cancer metastasis. Breast Cancer Res 15:R116. https://doi.org/10.1186/bcr3585.

38. Corte-Real S, Collins C, Aires da Silva F, Simas JP, Barbas CF, Chang Y, Moore P, Goncalves J. 2005. Intrabodies targeting the Kaposi sarcoma-associated herpesvirus latency antigen inhibit viral persistence in lymphoma cells. Blood 106:3797-3802. https://doi.org/10.1182/blood-2005-04-1627.

39. Obishakin E, Stijlemans B, Santi-Rocca J, Vandenberghe I, Devreese B Muldermans S, Bastin P, Magez S. 2014. Generation of a nanobody targeting the paraflagellar rod protein of trypanosomes. PLoS One 9:e115893. https://doi.org/10.1371/journal.pone.0115893.

40. Stijlemans B, Conrath K, Cortez-Retamozo V, Van Xong H, Wyns L, Senter P, Revets H, De Baetselier P, Muyldermans S, Magez S. 2004. Efficient targeting of conserved cryptic epitopes of infectious agents by single domain antibodies. African trypanosomes as paradigm. J Biol Chem 279: 1256-1261. https://doi.org/10.1074/jbc.M307341200.

41. Van Audenhove I, Van Impe K, Ruano-Gallego D, De Clercq S, De Muynck K, Vanloo B, Verstraete H, Fernández LÁ, Gettemans J. 2013. Mapping cytoskeletal protein function in cells by means of nanobodies. Cytoskeleton (Hoboken) 70:604-622. https://doi.org/10.1002/cm.21122.

42. Moutel S, Bery N, Bernard V, Keller L, Lemesre E, de Marco A, Ligat L, Rain J-C, Favre G, Olichon A, Perez F. 2016. NaLi-H1: a universal synthetic library of humanized nanobodies providing highly functional antibodies and intrabodies. Elife 5. https://doi.org/10.7554/eLife.16228.

43. Chirichella M, Lisi S, Fantini M, Goracci M, Calvello M, Brandi R, Arisi I, D’Onofrio M, Di Primio C, Cattaneo A. 2017. Post-translational selective intracellular silencing of acetylated proteins with de novo selected intrabodies. Nat Methods 14:279-282. https://doi.org/10.1038/nmeth.4144.

44. Abulrob A, Sprong $H$, Van Bergen En Henegouwen P, Stanimirovic D. 2005. The blood-brain barrier transmigrating single domain antibody: mechanisms of transport and antigenic epitopes in human brain endothelial cells. J Neurochem 95:1201-1214. https://doi.org/10.1111/j.1471-4159.2005.03463.x.

45. Stanimirovic D, Kemmerich K, Haqqani AS, Farrington GK. 2014. Engineering and pharmacology of blood-brain barrier-permeable bispecific antibodies. Adv Pharmacol 71:301-335. https://doi.org/10.1016/bs.apha.2014.06.005.

46. Matz H, Dooley H. 2019. Shark IgNAR-derived binding domains as potential diagnostic and therapeutic agents. Dev Comp Immunol 90:100-107. https://doi.org/10.1016/j.dci.2018.09.007.

47. Negi SS, Braun W. 2017. Cross-React: a new structural bioinformatics method for predicting allergen cross-reactivity. Bioinformatics 33:1014-1020. https://doi.org/10.1093/bioinformatics/btw767.

48. Faktorová $D$, Nisbet RER, Fernández Robledo JA, Casacuberta E, Sudek L, Allen $A E$, Ares $M$, Jr, Aresté $C$, Balestreri $C$, Barbrook $A C$, Beardslee $P$, Bender S, Booth DS, Bouget F-Y, Bowler C, Breglia SA, Brownlee C, Burger G, Cerutti H, Cesaroni R, Chiurillo MA, Clemente T, Coles DB, Collier JL, Cooney EC, Coyne K, Docampo R, Dupont CL, Edgcomb V, Einarsson E, Elustondo PA, Federici F, Freire-Beneitez V, Freyria NJ, Fukuda K, García PA, Girguis PR, Gomaa F, Gornik SG, Guo J, Hampl V, Hanawa Y, HaroContreras ER, Hehenberger E, Highfield A, Hirakawa Y, Hopes A, Howe CJ, Hu I, Ibañez J, et al. 2020. Genetic tool development in marine protists: emerging model organisms for experimental cell biology. Nat Methods 17:481-494. https://doi.org/10.1038/s41592-020-0796-x.

49. Bianchini P, Peres C, Oneto M, Galiani S, Vicidomini G, Diaspro A. 2015. STED nanoscopy: a glimpse into the future. Cell Tissue Res 360:143-150. https://doi.org/10.1007/s00441-015-2146-3.

50. Wheeler RJ, Gull K, Sunter JD. 2019. Coordination of the cell cycle in trypanosomes. Annu Rev Microbiol 73:133-154. https://doi.org/10.1146/annurev-micro -020518-115617.

51. Bastin P, Gull K. 1999. Assembly and function of complex flagellar structures illustrated by the paraflagellar rod of trypanosomes. Protist 150 : 113-123. https://doi.org/10.1016/S1434-4610(99)70015-5.

52. Dang HQ, Zhou Q, Rowlett VW, Hu H, Lee KJ, Margolin W, Li Z. 2017. Proximity interactions among basal body components in Trypanosoma brucei identify novel regulators of basal body biogenesis and inheritance. $\mathrm{mBio}$ 8. https://doi.org/10.1128/mBio.02120-16.

53. Woods J. 2019. Selection of functional intracellular nanobodies. SLAS Discov 24:703-713. https://doi.org/10.1177/2472555219853235.

54. Mir MA, Mehraj U, Sheikh BA, Hamdani SS. 2020. Nanobodies: the "magic bullets" in therapeutics, drug delivery and diagnostics. Human Antibodies 28:29-51. https://doi.org/10.3233/HAB-190390.

55. Pinto Torres JE, Goossens J, Ding J, Li Z, Lu S, Vertommen D, Naniima P, Chen R, Muyldermans S, Sterckx YG-J, Magez S. 2018. Development of a nanobody-based lateral flow assay to detect active Trypanosoma congolense infections. Sci Rep 8:9019. https://doi.org/10.1038/s41598-018-26732-7.

56. Manoutcharian K, Perez-Garmendia R, Gevorkian G. 2017. Recombinant antibody fragments for neurodegenerative diseases. Curr Neuropharmacol 15:779-788. https://doi.org/10.2174/1570159X01666160930121647.

57. Morrison C. 2019. Nanobody approval gives domain antibodies a boost. Nat Rev Drug Discov 18:485-487. https://doi.org/10.1038/d41573-019-00104-w.

58. De Vooght L, Caljon G, De Ridder K, Van Den Abbeele J. 2014. Delivery of a functional anti-trypanosome Nanobody in different tsetse fly tissues via a bacterial symbiont, Sodalis glossinidius. Microb Cell Fact 13:156. https:// doi.org/10.1186/s12934-014-0156-6.

59. Stijlemans B, Caljon G, Natesan SKA, Saerens D, Conrath K, Pérez-Morga D, Skepper JN, Nikolaou A, Brys L, Pays E, Magez S, Field MC, De Baetselier P, Muyldermans S. 2011. High affinity nanobodies against the Trypanosome brucei VSG are potent trypanolytic agents that block endocytosis. PLoS Pathog 7:e1002072. https://doi.org/10.1371/journal.ppat.1002072.

60. Wirtz E, Leal S, Ochatt C, Cross GA. 1999. A tightly regulated inducible expression system for conditional gene knock-outs and dominant-negative genetics in Trypanosoma brucei. Mol Biochem Parasitol 99:89-101. https://doi.org/10.1016/s0166-6851(99)00002-x.

61. Nossal NG, Heppel LA. 1966. The release of enzymes by osmotic shock from Escherichia coli in exponential phase. J Biol Chem 241:3055-3062. https://doi.org/10.1016/S0021-9258(18)96497-5.

62. Karlsson R, Katsamba PS, Nordin H, Pol E, Myszka DG. 2006. Analyzing a kinetic titration series using affinity biosensors. Anal Biochem 349:136-147. https://doi.org/10.1016/j.ab.2005.09.034.

63. Palau W, Di Primo C. 2012. Single-cycle kinetic analysis of ternary DNA complexes by surface plasmon resonance on a decaying surface. Biochimie 94:1891-1899. https://doi.org/10.1016/j.biochi.2012.04.025.

64. Heldin $\mathrm{CH}$, Johnsson A, Wennergren S, Wernstedt C, Betsholtz C, Westermark B. 1986. A human osteosarcoma cell line secretes a growth factor structurally related to a homodimer of PDGF A-chains. Nature 319:511-514. https://doi .org/10.1038/319511a0.

65. Hannaert V, Albert M-A, Rigden DJ, da Silva Giotto MT, Thiemann O, Garratt RC, Van Roy J, Opperdoes FR, Michels PAM. 2003. Kinetic characterization, structure modelling studies and crystallization of Trypanosoma brucei enolase. Eur J Biochem 270:3205-3213. https://doi.org/10.1046/j .1432-1033.2003.03692.x.

66. Woods A, Sherwin T, Sasse R, MacRae TH, Baines AJ, Gull K. 1989. Definition of individual components within the cytoskeleton of Trypanosoma brucei by a library of monoclonal antibodies. J Cell Science 93:491-500. https://doi .org/10.1242/jcs.93.3.491. 